\title{
LA MORTE DEL MONACO NELLE PIÙ ANTICHE FONTI FIGURATIVE BIZANTINE: DALLE ORIGINI AL SECOLO XI
}

\section{SIMONA MORETTI}

\section{UDC: 7.033 .2 $27-788$}

Original scientific paper

Manuscript received: 06. 11. 2016.

Revised manuscript accepted: 07. 02. 2017.

DOI: 10.1484/J.HAM.5.113746
S. Moretti

Università IULM

Facoltà di Arti, turismo e mercati

Dipartimento di Comunicazione, arti e media

Via Carlo Bo, 1 - 20143 Milano

Italia

This paper focuses on the theme of the Dormition of monks - hermits and/or ccenobites - as represented in the earliest figurative sources of Byzantine art, and especially in illustrated manuscripts. The study of the material led to the identification of some recurrent features, such as the sobriety of the composition and details, the inevitable monastic habit, the enhancement of humility and asceticism. An attempt has been made to create a dialogue between images and written sources, thanks to which a complex and vivid picture has emerged, reflecting multiple meanings that have to be analyzed through the eyes of the past. This paper also deals with the issue of the origin of this iconographic subject and its relationship with other models.

Keywords: Koimesis, Dormition, monk, nun, death, Byzantine art, humility.

A Maria, Cesare e la piccola Elisa

"Paolo, coperto di vilissima polvere, giace per risorgere alla gloria; voi, destinati a bruciare con le vostre ricchezze, siete oppressi dalle pietre di pesanti sepolcri. Risparmiate, vi prego, risparmiate almeno le ricchezze che amate! Perché anche i vostri morti avvolgete in vesti dorate? Perché l'ambizione non cessa tra i lutti e le lacrime? Forse i cadaveri dei ricchi non sanno marcire se non avvolti nella seta?". Così scriveva e domandava san Girolamo nella Vita di Paolo primo eremita, composta tra il 375 e il 377 e definita "prima opera monastica latina in assoluto"2. Questo contributo è dedicato alle immagini del monaco morto, giacente, nelle fonti figurative più antiche dell'Oriente greco fino all'XI secolo's.

All'iconografia della Dormizione ${ }^{4}$ dell'eremita ha dedicato un lavoro recente Manuela De Giorgi, prendendo in considerazione esempi bizantini e post-bizantini che vanno dal XIII al XVIII secolo e circoscrivendo lo studio alla produzione pittorica murale o su tavola ${ }^{5}$. In questa sede presteremo invece attenzione alle prime testimonianze visive note e alla

\footnotetext{
${ }^{1}$ Questo studio trae ispirazione da un vecchio lavoro, eseguito tra 2005 e 2007 e dedicato all'aggiornamento bibliografico della descrizione delle miniature del cosiddetto Menologio di Basilio II (Città del Vaticano, Biblioteca Apostolica Vaticana, Vat. gr. 1613) a partire dal 1907, anno di pubblicazione del primo facsimile del lussuoso manoscritto e del suo commentario. Ringrazio Antonio Iacobini che mi gettò nell'impresa e Francesco D’Aiuto che la agevolò. Il lavoro per diversi motivi non venne mai pubblicato, ma mi ha permesso di imparare molto. È merito di Gabriele Archetti avermi offerto lo stimolo a riprendere quelle ricerche così lontane nel tempo, e di Miljenko Jurković se questo studio vedrà la luce delle stampe; a loro due va la mia riconoscenza. Infine mi fa piacere ringraziare i relatori del convegno che, a vario titolo, e qualcuno anche inconsapevolmente, mi hanno indotto a meditare ulteriormente sul tema, tra questi: Nicola Busino, Giuseppe Motta, Meta Niederkorn-Bruck. E, last but not least, sono grata a Vera von Falkenhausen per un utile suggerimento. GIROLAMO, Vite degli eremiti Paolo, Ilarione e Malco, Introduzione, traduzione e note a cura di B. Degórski, Roma, 1996 (Collana di testi patristici, 126), p. 89. ${ }^{2}$ B. DEGÓRSKI, Introduzione, in Hieronymi historica et hagiographica. Vita Beati Pauli monachi Thebaei. Vita Hilarionis. Vita Malchi monachi captivi. Epistula praefatoria in Chronicis Eusebii Caesariensis. Chronicorum Eusebii Caesariensis continuatio. De viris inlustribus. In Regulae S. Pachomii versionem praefatio || Girolamo. Opere storiche e agiografiche. Vita di san Paolo, eremita di Tebe. Vita di Ilarione. Vita di Malco, l'eremita prigioniero. Prefazione alla traduzione delle Cronache di Eusebio di Cesarea. Continuazione delle Cronache di Eusebio di Cesarea. Gli uomini illustri. Prefazione alla traduzione della Regola di Pacomio, Roma, 2014 (Hieronymi opera, XV || Opere di Girolamo, XV), p. 15-42, in part. p. 21.

3 In generale, sulla morte a Bisanzio mi limito a rimandare a G.T. DENNIS, Death in Byzantium, in Dumbarton Oaks Papers, 55, 2001, p. 1-7. "Monks and nuns, in particular, were called to meditate on death. Typical is the exhortation by Symeon the New Theologian to his monks, 'On the Remembrance of Death'. The sixth rung on the Ladder of Paradise of John Klimakos bears the same title: «On the Remembrance of Death»” (Ibid., p. 1).

${ }^{4}$ Sull'uso di denominare la morte "dormizione" tra i primi cristiani vedi GIROLAMO, op. cit. (n. 1), n. 58 a p. 83.

${ }^{5}$ M. DE GIORGI, La dormizione dell'eremita, in A. Malquori (a cura di), con M. De Giorgi e L. Fenelli, Atlante delle Tebaidi e dei temi figurativi, Firenze, 2013, p. 189-199. Cfr., inoltre, le schede a seguire a firma di M. De Giorgi e A. Malquori (Ibid., p. 200-229). L'esempio più antico preso in considerazione dalla De Giorgi è l'icona, piuttosto mal ridotta, del Sinai con la Dormizione di un santo, forse Arsenio, databile al XIII secolo. Il quadro sintetico, così come è definito dall'autrice che pur sottolinea una completezza sufficiente, delle Koimeseis bizantine di eremiti è pubblicato alle p. 196-197. Già E. IOANNIDA-

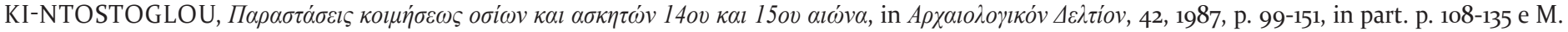

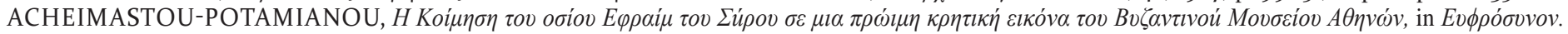

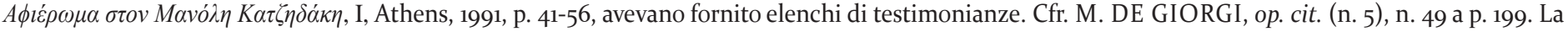
studiosa sottolinea la quasi omogeneità del gruppo: "Se si escludono infatti i primissimi esempi, la maggior parte di questo corpus, che conta ad oggi una trentina di manufatti, obbedisce a due criteri di massima: la loro pertinenza, in larga parte, alla scuola cretese, con una cronologia che va dalla seconda metà del Quattrocento fino al XVII secolo avanzato; e la scelta quasi univoca dei santi rappresentati che si concentra, sostanzialmente, su tre eremiti: Efrem il Siro, Onofrio e Saba" (Ibid., p. 196).

Per quanto riguarda più in generale il tema della morte nell'iconografia bizantina mi limito a menzionare: C. WALTER, Death in Byzantine Iconography, in Eastern Churches Review, 8, 1976, 2, p. 113-127, con 12 figg.; B. CVETKOVIĆ, The Living (and the) Dead Imagery of Death in Byzantium and the Balkans, in Ikon, 4, 2011, p. 27-44. Per un caso particolare vedi C. BORDINO, Le scene della morte di san Basilio e l'esaltazione della chiesa delle origini nelle pitture
} 


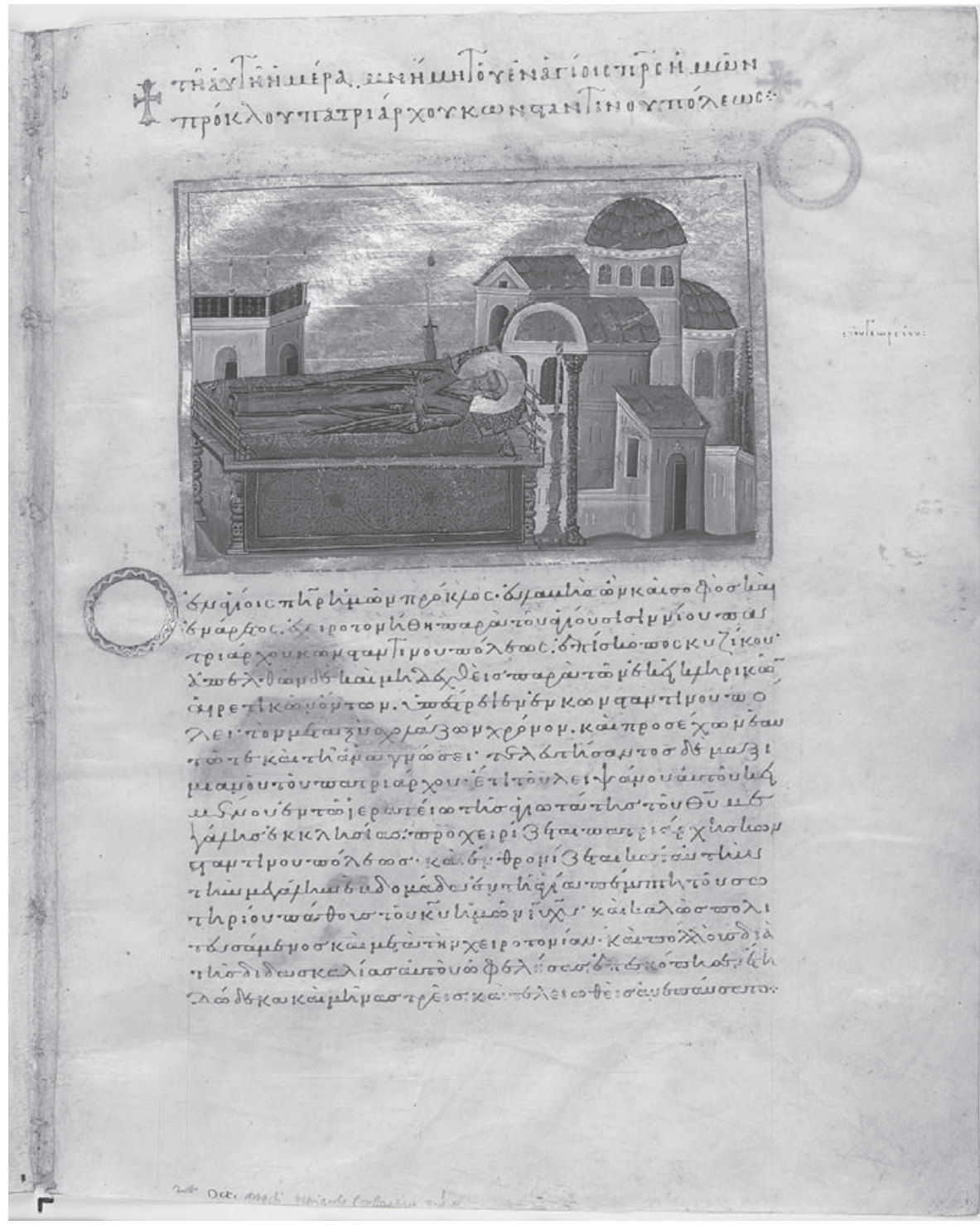

Fig. 1. Città del Vaticano, Biblioteca Apostolica Vaticana, Vat. gr. 1613, p. 136: Koimesis di san Proclo patriarca di Costantinopoli e sua notizia agiografica (@) 2017 per concessione della Biblioteca Apostolica Vaticana, ogni diritto riservato).

figura del monaco bizantino nella sua duplice 'veste', l'eremita e il cenobita, con uno sguardo privilegiato alla produzione miniata. L'analisi metterà in evidenza alcune caratteristiche ricorrenti come, ad esempio, la sobrietà della composizione, l'immancabileabbigliamento monastico, la valorizzazione dell'umiltà e dell'ascesi. La lettura delle fonti ci fornirà la spiegazione di dettagli iconografici e la conferma di consuetudini liturgiche.

\section{LE TESTIMONIANZE VISIVE}

La nostra indagine partirà da un testimone eccellente, il cosiddetto Menologio di Basilio II (Città del Vaticano, Biblioteca Apostolica Vaticana, Vat. gr. 1613), codice greco databile tra 979 e 1020 circa, ma più probabilmente dei primi decenni dell'XI secolo, che in realtà, come si sa, è un Sinassario riccamente miniato prodotto nello scriptorium delle Blacherne a Costantinopoli per l'imperatore Basilio II (976-1025) ${ }^{6}$. Il repertorio figurativo si deve a un'équipe di otto pittori e tra le immagini ispirate all'Antico e al Nuovo Testamento, ma anche a eventi storici, frequenti appaiono le scene di martirio, accompagnate da figure iconiche di santi e da pii personaggi rappresentati sul letto di morte.

Il manoscritto conserva solo i primi sei mesi del calendario liturgico bizantino, da settembre a febbraio, la seconda parte è perduta oppure non fu mai realizzata. Le miniature che corredano il testo sono ben quattrocentotrenta e solo sette hanno come soggetto principale la Koimesis del monaco o della monaca (escludendo i personaggi che, pur avendo scelto all'inizio la vita monastica, fecero poi una brillante carriera all'interno della Chiesa e divennero vescovi). Le Dormizioni illustrate nel codice vaticano possono mostrare al lettore, talvolta, cadaveri deposti su ricchi letti funebri, eventualmente accompagnati da candele, ma non si tratta mai di santi personaggi che vissero e morirono da monaci, senza mai accettare cariche importanti, fig. 1)7.

del X secolo in Cappadocia, in N. Bock, I. Foletti, M. Tomasi (sous la dir.), L'évêque, l'image et la mort. Identité et mémoire au Moyen Âge, Roma, 2014 (Études lausannoises d'histoire de l'art, 16), p. 533-549. R. Korsova sottolinea come nell'arte bizantina san Basilio sia considerato non tanto monaco, quanto piuttosto vescovo, nelle cui vesti è effigiato, ad esempio, nel cod. gr. 510, f. 104r, della Bibliothèque nationale de France (databile tra l'879 e l'882), dove è rappresentata la processione funeraria; anche sul Menologio di Basilio II, Vat. gr. 1613, p. 288, san Basilio, raffigurato stante in tutta la sua autorevolezza, è in abiti vescovili. Cfr. R. KORSOVA, A Tenth-Century Graffito of St. Basil the Great in the light of his Cult in Eastern Monasticism, in Palaeobulgarica, 22, 1, 1998, p. 75-95, in part. p. 75, 88.

${ }^{6} \mathrm{Su}$ questo illustre testimone mi limito a rimandare al volume di commento che accompagna il recente facsimile del manoscritto: F. D'Aiuto (dirigido por), I. Pérez Martín (edición española a cargo de), El «Menologio de Basilio II», Città del Vaticano, Biblioteca Apostolica Vaticana, Vat. gr. 1613, Libro de estudios con ocasión de la edición facsímil, Città del Vaticano-Atenas-Madrid, 2008 (Colección Scriptorium, 18). Su Basilio II e la produzione artistica vedi L. RICCARDI, «Un altro cielo»: l'imperatore Basilio II e le arti, in Rivista dell'Istituto Nazionale d'Archeologia e Storia dell'arte, 61, terza serie, XXIX, 2006 (ma 2011), p. 103-145 (per il manoscritto vaticano: p. 107-110).

7 Elenco qui le scene legate ai funerali di un santo, non necessariamente monaco, rappresentate nel codice (includo: l'esposizione del corpo - che corrisponde in maniera stringente all'iconografia che definiamo Koimesis -, la processione funebre, il seppellimento; escludo: le processioni al sepolcro in occasione delle commemorazioni, invenzioni e traslazioni successive di corpi): Vat. gr. 1613, p. 3 (Giosuè: viene sepolto in un sarcofago), 13 (Mosè: Michele arcangelo depone il suo corpo ai piedi di un'altura), 15 (san Abdia martire: i suoi carnefici lo trasportano su un letto riccamente ornato), 43 (sante Pistis, Elpis e Agape martiri: la madre depone le loro spoglie in un unico sarcofago), 45 (santi Geminiano e Lucia: quest'ultima muore in pace), 52 (santa Susanna martire in Palestina: vedi qui testo e fig. 2), 83 (san Adautto martire: la consorte e la figlia lo seppelliscono in un sarcofago), 9o (sant'Amun abate: vedi qui testo e fig. 3), 121 (san Luca evangelista: deposizione nel sarcofago, compare un personaggio con incensiere presso il suo capo), 125 (san Cornelio centurione: il suo corpo è su un ricco catafalco, tiene sul petto il Vangelo), 134 (sant'Ignazio patriarca di Costantinopoli: la sua salma, adagiata su coperta e cuscino splendenti, è esposta davanti al monastero di Satyro dove verrà sepolto), 136 (san Proclo patriarca di Costantinopoli: il suo corpo è su un lussuoso cataletto, appaiono candele accese, fig. 1), 154 (santi Giovanni e Giacomo Zelotes: deposizione delle loro salme in un unico sarcofago, un ecclesiastico agita al loro capo un turibolo), 186 (san Matteo apostolo ed evangelista: è deposto in una semplice bara, un religioso al suo capo tiene l'incensiere), 210 (santi 
Osserviamo rapidamente i sette casi, prestando progressivamente attenzione ad alcuni aspetti.

\section{LA SOBRIETÀ DELLA RAPPRESENTAZIONE}

Santa Susanna (Vat. gr. 1613, p. 52$)^{8}$ è effigiata defunta nel carcere di Eleuteropoli (fig. 2). Originaria della Palestina, era figlia di Artemio, un sacerdote pagano; rimasta orfana di entrambi i genitori, venne istruita da un prete di nome Silvano e, battezzata, donò tutti i suoi beni ed entrò in monastero con il nome di Giovanni. Accusata da una donna di avere avuto rapporti sessuali con lei, dovette svelare la sua vera identità. Lasciò dunque la comunità e si recò a Eleuteropoli, qui venne ordinata diaconessa dal vescovo del luogo, ma dal momento che predicava la parola del Signore fu portata davanti al prefetto, atrocemente torturata e rinchiusa in carcere, dove mori ${ }^{9}$. Visse forse nel IV secolo ${ }^{10}$.

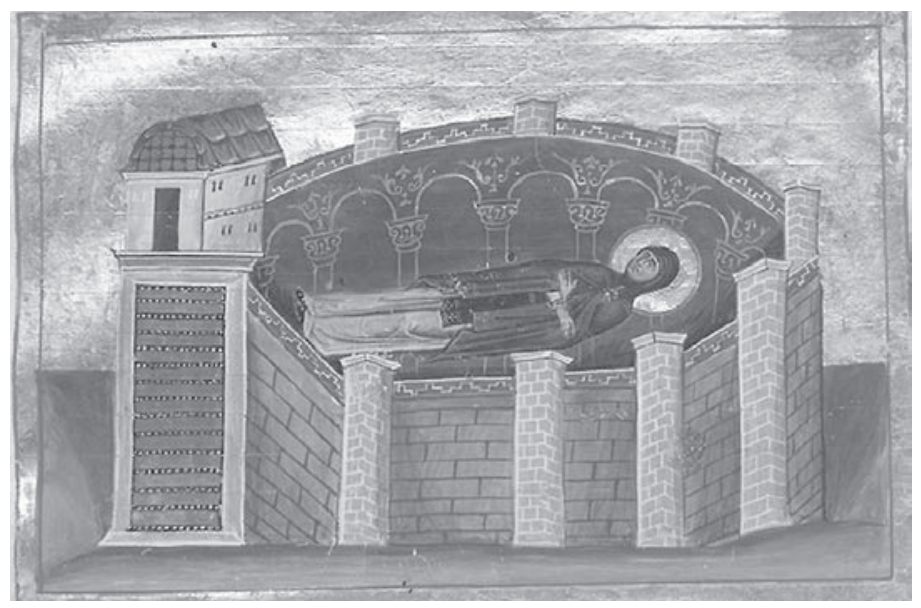

Fig. 2. Città del Vaticano, Biblioteca Apostolica Vaticana, Vat. gr. 1613, p. 52: Koimesis di santa Susanna (C) 2017 per concessione della Biblioteca Apostolica Vaticana, ogni diritto riservato).
Sant'Amun abate (Vat. gr. 1613, p. 90) ${ }^{11}$, eremita d'Egitto nel IV secolo, è rappresentato morto ${ }^{12}$ e disteso sopra un umile graticcio (fig. 3), e in effetti le fonti raccontano che i monaci solevano riposare sopra una semplice stuoia ${ }^{13}$ e volevano essere deposti sul rozzo saio prima di morire ${ }^{14}$. Al capo dell'anziano Amun un vecchio monaco agita un turibolo per incensare il defunto, dietro di esso sta un altro monaco che si appoggia ad un bastone, entrambi hanno un'aria severa. All'estrema destra compare anche Antonio abate, appoggiato al bastone con il manico piegato ad angolo retto ${ }^{15}$. A lui fu concessa una visione straordinaria, rappresentata nella miniatura: egli alza lo sguardo al cielo e fa un gesto di stupore, in alto infatti si leva, accompagnata da due angeli, l'anima di Amun ${ }^{16}$. Di questa visione si legge nella Vita di Antonio scritta da Atanasio (295 circa-373) all'indomani della morte di Antonio avvenuta all'inizio del $356^{17}$, nella Storia Lausiaca

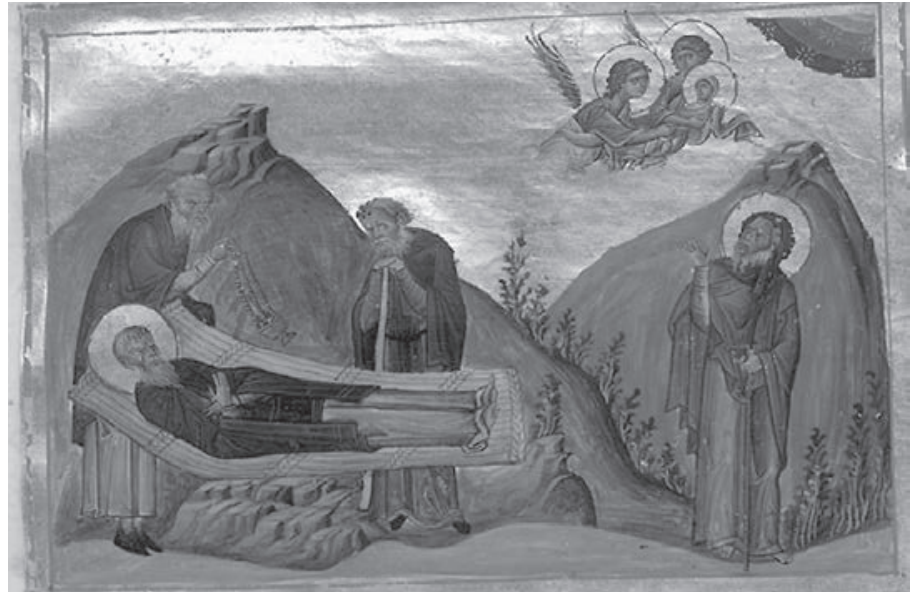

Fig. 3. Città del Vaticano, Biblioteca Apostolica Vaticana, Vat. gr. 1613, p. 90: Koimesis di sant'Amum abate (C) 2017 per concessione della Biblioteca Apostolica Vaticana, ogni diritto riservato).

Stefano iuniore, Pietro e Andrea martiri: il monaco Pietro giace disteso e sembra apparentemente già morto; non includo questo caso tra quelli esaminati nel dettaglio perché qui abbiamo una scena di martirio in cui la figura di Pietro defunto ha semplice funzione di dettaglio), 218 (san Filareto elemosiniere: è deposto in un sarcofago, un ecclesiastico a lato del suo capo lo incensa), 294 (san Teagene vescovo e martire: è deposto in un sarcofago e ha il Vangelo sul petto, un ecclesiastico a lato del suo capo lo incensa), 298 (san Michea profeta: è deposto in un'umile bara), 321 (san Paolo primo eremita: vedi qui testo e fig. 4), 327 (sant'Antonio abate: vedi qui testo e fig. 5), 330 (sant'Eusebia romana: vedi qui testo e fig. 6), 341 (san Timoteo apostolo e vescovo di Efeso: trasporto delle sue spoglie raccolte in un sarcofago verso una chiesa con cupole, compaiono candele e incensiere), 344 (Traslazione delle reliquie di sant'Anastasio Persiano, chiuse in una bara, appaiono candele e turibolo), 354 (sant'Efrem siro: vedi qui testo e fig. 7), 394 (santa Marina: vedi qui testo e fig. 9), 406 (san Maruta e deposizione delle reliquie dei martiri persiani in Martiropoli: il sarcofago è deposto davanti una chiesa, compaiono candele e l'immancabile incensiere).

${ }^{8}$ Commemorata il 20 settembre. Per una sintetica descrizione della miniatura: P. Franchi De' Cavalieri (a cura di), Il Menologio di Basilio II (cod. Vaticano greco 1613), I, Torino, 1907 (Codices e Vaticanis selecti phototypice expressi, 8), p. 16.

${ }_{9}^{9}$ Le brevi notizie che riporto si possono leggere sul manoscritto vaticano.

${ }^{10}$ J. M. SAUGET, s.v., Susanna, vergine, santa, martire di Eleuteropoli, in Bibliotheca Sanctorum, XII, Roma, 1969, col. 77.

${ }^{1 "}$ Commemorato il 4 ottobre. Per una descrizione sintetica della miniatura: P. Franchi De' Cavalieri (a cura di), op. cit. (n. 8), p. $25-26$.

${ }^{12}$ Morì prima di Antonio abate, quindi prima del 17 gennaio 356, infatti quest'ultimo, nel suo eremo, vide la sua anima portata in cielo da angeli (vedi più avanti nel testo): M. SALSANO, s.v., Ammonio (Ammon, Ammun, Amun), anacoreta di Nitria, santo, in Bibliotheca Sanctorum, I, Roma, 1961, col. 1014-1015. ${ }^{13}$ Ad esempio, leggiamo in GIROLAMO, op. cit. (n. 1), p. 100 (4.2), che Ilarione "Dormì fino alla morte sulla nuda terra e su un giaciglio di giunchi".

${ }^{14}$ Ibidem, n. 27 a p. 96.

${ }^{15}$ Sul bastone mi limito a menzionare GIOVANNI CASSIANO, Le istituzioni cenobitiche, Introduzione e traduzione a cura di L. Dattrino, Vicenza, 1989 (Scritti monastici, 13), p. 64 (Libro I.8: "Il bastone è il simbolo dell'energia necessaria per respingere le tentazioni"). L'autore, vissuto tra IV e V secolo, "discepolo in Oriente e maestro in Occidente" come è stato definito (L. DATTRINO, Introduzione, Ibid., p. 5-48, in part. p. 46), nei primi libri delle Istituzioni monastiche sembra "offrire anzitutto una descrizione della vita condotta nei monasteri dell'Egitto e della Palestina da lui visitati" (Ibid., p. 27).

${ }^{16}$ Sull'anima nella letteratura patristica e bizantina vedi in generale: N. CONSTAS, "To Sleep, Perchance to Dream": The Middle State of Souls in Patristic and Byzantine Literature, in Dumbarton Oaks Papers, 55, 2001, p. 91-123. L'autore menziona anche questa miniatura: Ibid., n. 53 a p. 106. Cfr. per la rappresentazione dell'anima nell'arte bizantina almeno B. CVETKOVIĆ, op. cit. (n. 5), p. 28 e ss. Per l'illustrazione del "Kanon eis Psychorragounta” vedi V. MARINIS, "He Who Is at the Point of Death": The Fate of the Soul in Byzantine Art and Liturgy, in Gesta, 54, 1, 2015, p. 59-84. Per la sorte dell'anima nelle Vite dei saloi Simeone e Andrea, con riferimento alla visuality, cfr. P. CESARETTI, B. HAMARNEH, Testo agiografico e orizzonte visivo. Ricontestualizzare le Vite dei saloi Simeone e Andrea (BHG 1677, 115z), Premessa di A. Acconcia Longo, Roma 2016 (Testi e Studi Bizantino-Neoellenici, XX), p. 108-111.

${ }^{17}$ ATANASIO DI ALESSANDRIA, Vita di Antonio, Traduzione, introduzione e note a cura di D. Baldi, Roma, 2015 (Collana di testi patristici, 241), p. 96-97 (6o.1-3). Cfr. D. BALDI, Introduzione, Ibid., p. 5-27, in part. n. 4 alle p. 6-7; e inoltre P. GEMEINHARDT, Antonio. Il monaco che visse nel deserto, Bologna, 2015 (ed. or. München, 2013), p. 73. 


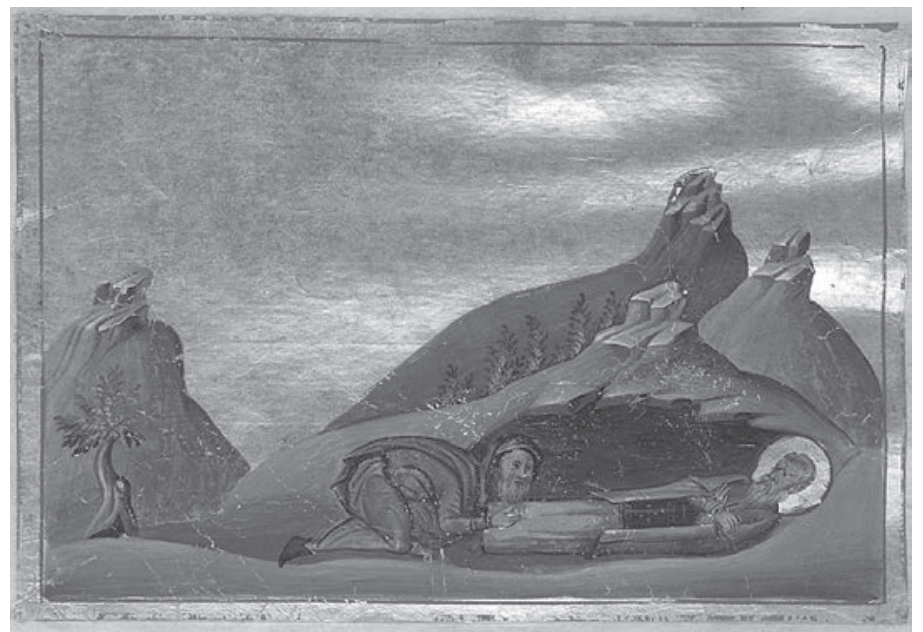

Fig. 4. Città del Vaticano, Biblioteca Apostolica Vaticana, Vat. gr. 1613, p. 321: Koimesis di san Paolo primo eremita (C) 2017 per concessione della Biblioteca Apostolica Vaticana, ogni diritto riservato).

di Palladio (419-420) ${ }^{18}$ e nella notizia agiografica che accompagna la miniatura sul manoscritto vaticano ${ }^{19}$. E di fatto un leitmotif nella letteratura agiografica e monastica, per la facoltà concessa agli asceti di vedere gli avvenimenti da lontano ${ }^{20}$. La scena è allestita all'aperto, dietro i personaggi due monti sono disegnati all'orizzonte, un po' di vegetazione compare sul terreno e sulle alture.

San Paolo primo eremita (Vat. gr. 1613, p. 321) ${ }^{21}$, nato nel 228 e morto nel 341, qualora sia realmente esistito ${ }^{22}$, giace cadavere davanti a una grotta (fig. 4). È vecchio, la lunga barba e i capelli sono bianchi. Nella sua Vita, scritta da Girolamo, leggiamo che il beato Antonio trovò il cadavere del santo, lo portò fuori dalla spelonca e cantò anche i salmi secondo la tradizione cristiana, lo tumulò in una fossa semplice scavata da due leoni ${ }^{23}$.

Dai Racconti del Sinai di Anastasio, composti verso il 675, apprendiamo che i monaci della Santa Montagna, una volta defunti, potevano essere tumulati in una fossa comune op-

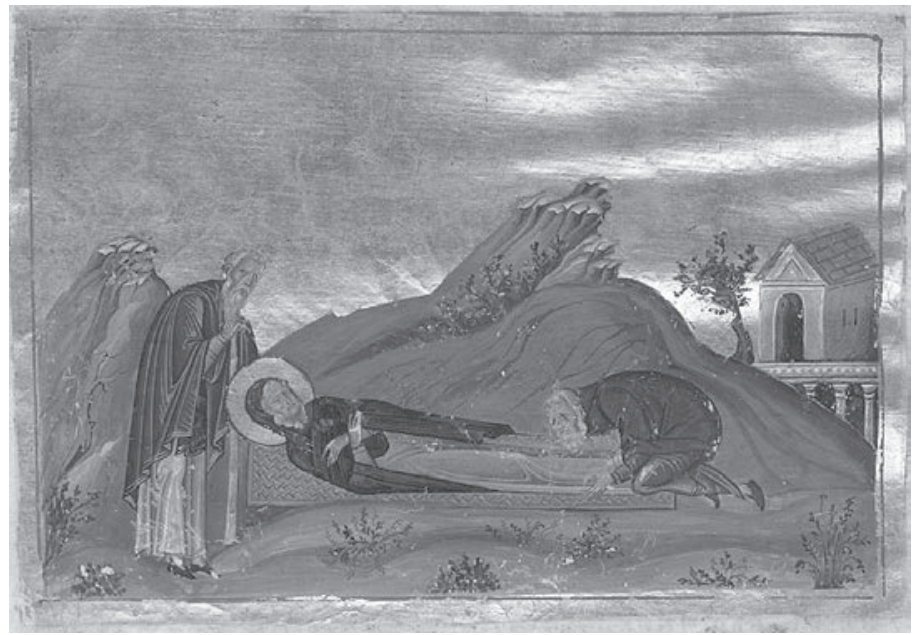

Fig. 5. Città del Vaticano, Biblioteca Apostolica Vaticana, Vat. gr. 1613, p. 327: Koimesis di sant'Antonio abate (C) 2017 per concessione della Biblioteca Apostolica Vaticana, ogni diritto riservato).

pure in una cella eremitica o in una caverna vicina alla cella ${ }^{24}$.

Nella miniatura del codice vaticano, ai piedi di san Paolo c'è Antonio, privo di nimbo e in proskynesis, fortemente restaurato in epoca moderna ${ }^{25}$. Girolamo racconta che Antonio, giunto alla grotta di Paolo, non capisce subito che questo è morto e lo crede in preghiera "ma poi, non percependo i sospiri che l'orante era solito emettere, chinatosi in lieve bacio, comprese che anche il cadavere del santo pregava Dio" ${ }^{26}$; ebbe pure, prima di arrivare alla spelonca del santo, la visione dell'anima portata in cielo dagli angeli ${ }^{27}$, miracolo che però non è narrato nel testo del codice vaticano e non è raffigurato nell'illustrazione che l'accompagna. La scena in questa miniatura è collocata in un paesaggio montuoso con poca vegetazione.

Sant'Antonio abate (Vat. gr. 1613, p. 327) ${ }^{28}$, nato in Egitto nel 251 e morto probabilmente nel $356^{29}$, è disteso senza vita su una stuoia gialla posizionata a terra (fig. 5). Si prostra ai suoi piedi un vecchio monaco dal volto triste ${ }^{30}$ per baciarlo,

${ }^{18}$ PALLADIO, La Storia Lausiaca, Introduzione di Ch. Mohrmann, Testo critico e commento a cura di G.J.M. Bartelink, Traduzione di M. Barchiesi, Milano, 1990 (Scrittori greci e latini; Ia ed. 1974), p. 40-41 (7.6), 44-45 (8.6).

19 Vat. gr. 1613, p. 90.

${ }^{20}$ GIROLAMO, op. cit. (n. 1), n. 136 alle p. 126-127.

${ }^{21}$ Commemorato il 15 gennaio. Per una descrizione sintetica della miniatura: P. Franchi De’ Cavalieri (a cura di), op. cit. (n. 8), p. 87.

${ }^{22}$ G. CALIÒ, A. CARDINALI, s.v., Paolo di Tebe, eremita, santo, in Bibliotheca Sanctorum, X, Roma, 1968, col. 269-280; E. WIPSZYCKA, s.v., Paolo di Tebe (IV sec.), in Nuovo dizionario patristico e di antichità cristiane, III, P-Z, Genova-Milano, 2008, col. 3864-3866.

${ }^{23}$ Per una traduzione italiana della Vita cfr. GIROLAMO, op. cit. (n. 1), p. 63-89, in part. sulla morte e tumulazione di Paolo p. 86-88 (14-16). L'omaggio di Antonio a Paolo (ne cerca l'incontro e poi gli dà sepoltura) non è riportato nella Vita di Antonio: B. DEGÓRSKI, Introduzione, in GIROLAMO, op. cit. (n. 1), p. 7-44, in part. p. 17 .

${ }^{24}$ R.M. PARRINELLO, Introduzione, I. Giovanni Climaco e il monachesimo sinaita, in GIOVANNI CLIMACO, La scala del paradiso, Introduzione, traduzione e note di R.M. Parrinello, Milano, 2007 (Letterature cristiane del primo millennio, 41), p. 9-48, in part. p. 45-46. Cfr. anche p. 34 e 39 . Sui rituali di sepoltura a Bisanzio cfr. almeno N. PATTERSON ŠEVČENKO, C.S. SNIVELI, D. ABRAHAMSE, N.B. TETERIATNIKOV, S. ĆURČIĆ, Ritual of Death in the Middle Byzantine Period, in Greek Orthodox Theological Review, 29, 2, 1984, p. 115-195; N. TETERIATNIKOV, A. KARPOZILOS, s.V., Burial, in The Oxford Dictonary of Byzantium, I, New York-Oxford, 1991, p. 340-341; A. KARPOZILOS, A. KAZHDAN, N. TETERIATNIKOV, A. CUTLER, s.v., Funeral, Ibid., II, p. 808-809; B. CVETKOVIĆ, op. cit. (n. 5); S.V. MOORE, 11. Experiencing Mid-Byzantine Mortuary Practice: Shrouding the Dead, in C. Nesbitt, M. Jackson (ed.), Experiencing Byzantium: Papers from the 44th Spring Symposium of Byzantine Studies (Newcastle and Durham, April 2011), FarnhamBurlington, 2013 (Society for the Promotion of Byzantine Studies. Publications, 18), p. 195-210.

${ }^{25}$ Sui restauri settecenteschi alle miniature del Menologio vaticano, che qui non commenterò, si veda in ultimo A. ZACHAROVA, Las restauraciones de las miniaturas en el siglo XVIII: descripción de las intervenciones, in F. D’Aiuto (dirigido por), I. Pérez Martín (edición española a cargo de), El «Menologio de Basilio II», op. cit. (n. 6), p. 299-305.

${ }^{26}$ GIROLAMO, op. cit. (n. 1), p. 86 (15).

${ }^{27}$ Ibidem, p. 86 (14).

${ }^{28}$ Commemorato il 17 gennaio. Per una descrizione sintetica della miniatura: P. FRANCHI DE' CAVALIERI, op. cit. (n. 8), p. 88-89.

${ }^{29}$ F. CARAFFA, A. RIGOLI, M. CIRMENI BOSI, s.v., Antonio, Abate, santo, in Bibliotheca Sanctorum, II, Roma, 1962, col. 106-136; T. ORLANDI, s.v., Antonio abate, in Nuovo dizionario patristico e di antichità cristiane, I, A-E, Genova-Milano, 2006, col. 369-370.

${ }^{30}$ Sulla rappresentazione degli affetti a Bisanzio mi limito a rimandare ai recenti contributi di V. DEL RE, L'iconografia delle quattro donne piangenti. I casi di Aquileia, Sant'Angelo in Formis, Venezia, tra anomalie iconografiche e trasmissione di modelli, in V. Camelliti, A. Trivellone (a cura di), Un Medioevo 
secondo l'ossequio del bacio che si faceva ai morti ${ }^{31}$, mentre un altro, sempre anziano, sta dritto presso la testa appoggiandosi a un bastone. Nella sua Vita scritta da Atanasio leggiamo che egli chiese, in punto di morte, a due confratelli di essere sepolto in un luogo noto solo ad alcuni di loro ${ }^{32}$; nella Vita dell'eremita Paolo redatta da Girolamo leggiamo che Amata diede sepoltura al corpo del diletto maestro33; lo stesso autore nella Vita di Ilarione racconta che quest'ultimo chiese a Isacco e Pelusiano, due discepoli di sant'Antonio, di mostrargli "il luogo dove si trovava la tomba di Antonio. Essi lo condussero in disparte, ma si ignora se gliela abbiano mostrata o meno, riferendo che, conformemente all'ordine di Antonio, questa segretezza era data affinché Pergamio, il quale era l'uomo più ricco del luogo, non traslasse nella propria villa il corpo del santo e non erigesse un martirio"34; nella Storia Lausiaca sono i discepoli Macario e Amata a seppellire il corpo del centenario Antonio ${ }^{35}$. Nel testo vaticano è riportata la notizia che due disce-

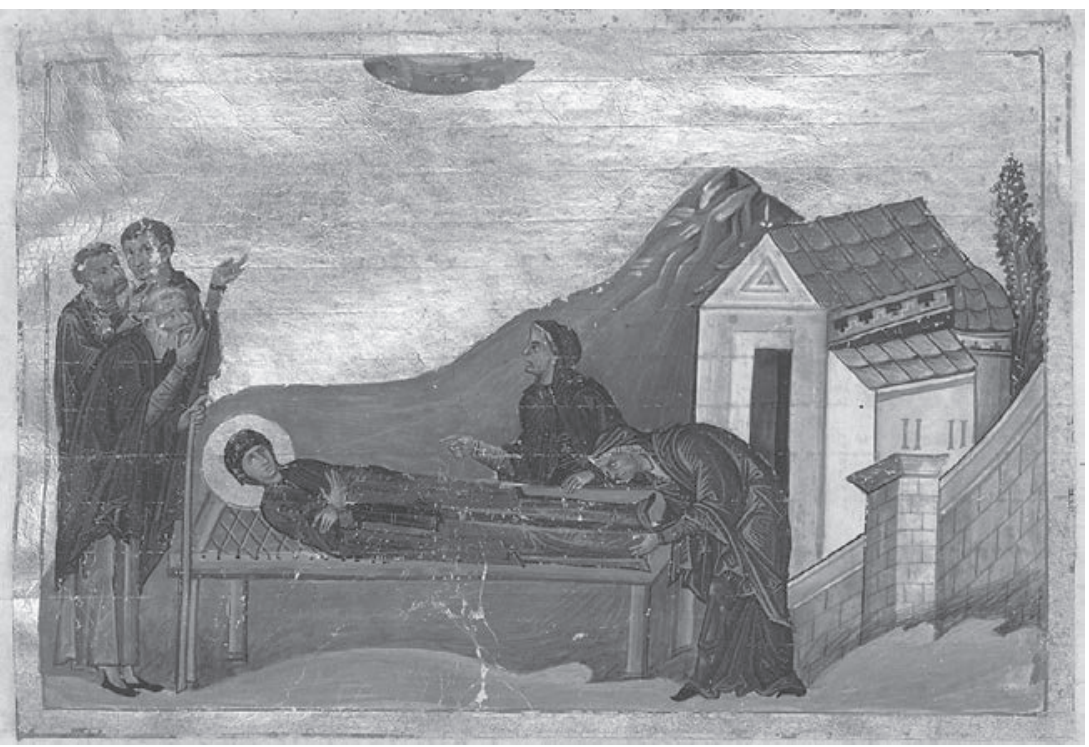
poli - di cui non è dichiarato il nome - lo tumularono in un luogo ignoto ${ }^{36}$. All'interrogativo perché non vi fu alcuna tomba per Antonio è stato risposto in maniera diversa e probabilmente ogni spiegazione contiene un po' di verità 37 , qui val la pena rileggere cosa scrive il suo biografo in merito. "Questa fu la conclusione della vita corporale di Antonio e quella fu l'inizio dell'ascesi" ${ }^{8}$ : dunque l'ascesi, alla fine dei conti, era ciò che importava. Di nuovo, nella miniatura, la scena è ambientata all'aperto e pochi elementi caratterizzano il paesaggio (alcune alture, un po' di vegetazione - qui compaiono addirittura fiori rossi -, un edificio con colonnato).

Santa Eusebia romana (Vat. gr. 1613, p. 330) ${ }^{39}$, vissuta forse nel $\mathrm{V}$ secolo ${ }^{40} \mathrm{o}$ tra $\mathrm{VeVI}^{41}$, giace cadavere su un povero letto, consistente in un'intelaiatura di legno, provvista di

una rete di corda (fig. 6) ${ }^{42}$. Ai piedi del letto sono le sue due ancelle: una s'inchina probabilmente per baciarle i piedi, l'altra indica la salma a un gruppo di tre monaci che si trova a capo del letto. Uno di loro, il più anziano, solleva leggermente il braccio e la mano destra eguarda verso l'alto, mentre con la sinistra stringe un bastone leggermente ricurvo alla sommità ${ }^{43}$. Anche gli altri due giovani monaci indirizzano la loro attenzione verso il cielo dove, secondo il Sinassario di Costantinopoli e la versione del Metafrasta, dovrebbe comparire una croce: questa miracolosa visione che ebbe luogo dopo la morte di Eusebia, tuttavia non è riportata nel testo vaticano pur essendo sottintesa dall'immagine ${ }^{44}$. È questo un caso in cui illustrazione e notizia agiografica non trovano corrispondenza: probabilmente il miniatore copia

in lungo e in largo. Da Bisanzio all'occidente (VI-XVI secolo). Studi per Valentino Pace, Pisa, 2014, p. 93-103; V. PACE, Natura e figura della "maniera greca" nella storiografia italiana e nella realtà, in A.C. Quintavalle (a cura di), Il Medioevo. Natura e figura. La raffigurazione dell'uomo e della natura nell'arte medievale, Atti del convegno internazionale di studi (Parma, 20-25 settembre 2011), Milano, 2015 (I convegni di Parma, 14), p. 745-753, in part. p. 749 e ss. ${ }^{31}$ G.T. DENNIS, op. cit. (n. 3), p. 3: "The blissful deaths of saintly monks and nuns, often surrounded by their disciples, conclude many a vita". Sull'uso e il significato del bacio nei primi secoli del cristianesimo rimando alla bibliografia, un po' datata ma ancora valida, menzionata in GIROLAMO, op. cit. (n. 1), n. 54 alle p. 81-82.

${ }^{32}$ ATANASIO DI ALESSANDRIA, op. cit. (n. 17), in part. sulla morte di Antonio p. 122-126 (89-93). Cfr. M. ALEXANDRE, À propos du récit de la mort d'Antoine (Athanase, Vie d'Antoine. PG 26, 968-974, \$ 89-93). L'heure de la mort dans la littérature monastique, in Le temps chrétien de la fin de l'Antiquité au Moyen Âge III ${ }^{e}$-XIII siècles, Actes du Colloques (Paris, École Normale Supérieure, 9-12 mars 1981), sous la responsabilité de Monsieur J.-M. Leroux, Paris, 1984, p. 263-282. Atanasio scrive che la morte di Antonio è degna di emulazione (Ibid., p. 263): ATANASIO DI ALESSANDRIA, op. cit. (n. 17), p. 122 (89.1). 33 GIROLAMO, op. cit. (n. 1), p. 64 (1).

34 Ibidem, p. 132 (21.9-10)

${ }_{35}$ PALLADIO, op. cit. (n. 18), p. 104-107 (21.1). Antonio visse 105 anni, ma nel Menologio di Basilio II si legge che morì a 95 anni (Vat. gr. 1613, p. 327).

${ }^{36}$ Vat. gr. 1613, p. 327.

${ }^{37}$ P. GEMEINHARDT, op. cit. (n. 17), p. 126-127.

${ }^{38}$ ATANASIO DI ALESSANDRIA, op. cit. (n. 17), p. 125 (93.1).

${ }^{39}$ Commemorata il 18 gennaio. Per una descrizione sintetica della miniatura: P. FRANCHI DE' CAVALIERI, op. cit. (n. 8), p. 89.

${ }^{40}$ C. DE CLERCQ, s.v., Eusebia di Milasa, santa, in Bibliotheca Sanctorum, V, Roma, 1964, col. 245.

${ }^{41}$ V. RUGGIERI, A. ACCONCI, J.M. FEATHERSTONE, Amboni carî e la «Vita Xenae seu Eusebiae» di Mylasa, in Orientalia Christiana Periodica, 68, 1, 2002, p. 37-88, in part. p. 40 e 69 .

${ }^{42}$ M.G. PARANI, Reconstructing the Reality of Images. Byzantine Material Culture and Religious Iconography (11 ${ }^{\text {th }}-15^{\text {th }}$ Centuries), Leiden-Boston, 2003 (The Medieval Mediterranean. Peoples, Economies and Cultures, 400-1453, 41), p. 177 e n. 101: la studiosa procedendo in un'analisi della cultura materiale bizantina attraverso le testimonianze artistiche dall'XI al XV secolo, con il supporto delle fonti scritte, nell'affrontare l'immagine del letto riscontra che sul Menologio di Basilio II troviamo la raffigurazione di un tipo molto semplice, visibile proprio in questa miniatura e su cui giace la santa monaca deceduta. ${ }^{43}$ P. FRANCHI DE' CAVALIERI, op. cit. (n. 8), p. 89: è probabilmente Paolo, che aveva trovato un alloggio per Eusebia presso la chiesa di Santo Stefano a Milasa.

${ }_{44}^{4}$ N. PATTERSON ŠEVČENKO, The Walters “Imperial" Menologium, in The Journal of the Walters Art Gallery, 51, 1993, p. 43-64, in part. p. 56-57, figg. 5051. La studiosa sottolinea la ripresa di questa iconografia nel Menologio Walters (Baltimora, Walters Art Gallery, W. 521, f. 228r), ma registra una differenza sulla data: la santa è commemorata nel Vat. gr. 1613 il 18 gennaio e non il 24 come nel manoscritto di Baltimora. Inoltre, Patterson Ševčenko fa notare la 


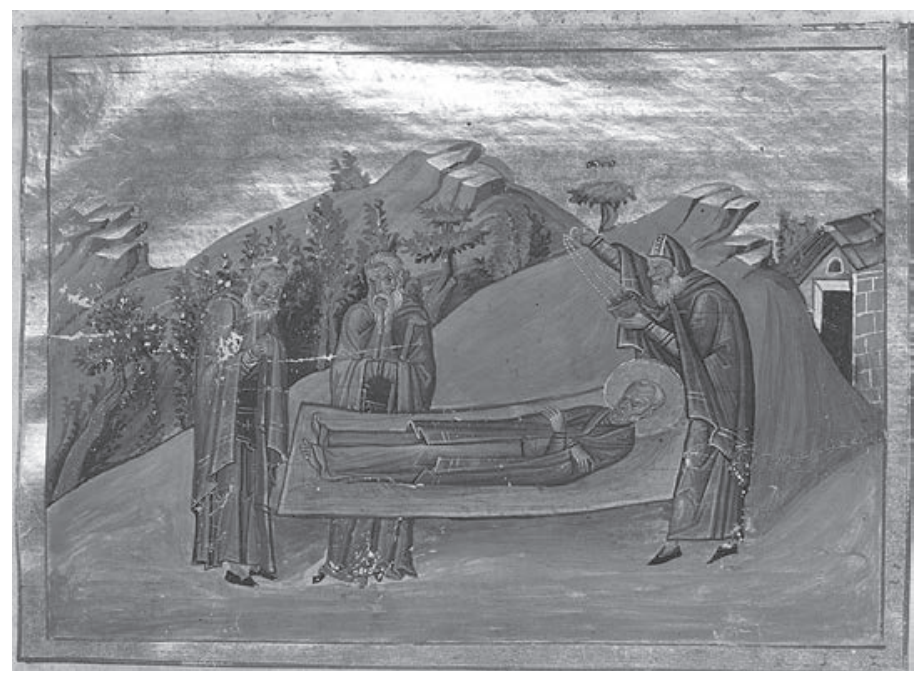

Fig. 7. Città del Vaticano, Biblioteca Apostolica Vaticana, Vat. gr. 1613, p. 354: Koimesis di sant'Efrem siro (2017 per concessione della Biblioteca Apostolica Vaticana, ogni diritto riservato).

un modello che non comprende ${ }^{45}$. La scena è rappresentata all'aperto, l'edificio sul fondo potrebbe essere, secondo Pio Franchi de' Cavalieri, la chiesa di Santo Stefano a Milasa, in Asia Minore, presso la quale Eusebia (anche detta Xena) trovò riparo ${ }^{46}$ oppure il luogo dove fu sepolta ${ }^{47}$. Il cadavere di "questa signora romana che attraversa il Mediterraneo per approdare, infine, sulle coste carie" venne posto, leggiamo nel suo bios, "nella piazza creatasi lungo la strada principale = Mese, dove avviene la veglia notturna; la mattina, dopo la liturgia funebre all'aperto, salmodiando e in processione, si

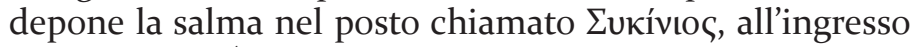
sud della città (questo posto era stato voluto da Xena, senza, tuttavia, che nel testo vi sia un accenno né un indizio che questo sito fosse sacro o monastico o funerario o acquistato in precedenza dalla Santa)" ${ }^{3}$. Nella miniatura è raffigurata la prothesis del corpo e non una processione funebre ${ }^{49}$, sebbene il luogo non sembri una plateia e l'edificio sullo sfondo possa evocare, come già detto, proprio la tomba di Eusebia.

Sant'Efrem siro (Vat. gr. 1613, p. 354) ) $^{50}$ ormai magro cadavere, è disteso sopra una stuoia (fig. 7). Al suo capo, canuto e con poca barba, sta un anziano monaco intento a muovere un turibolo, altri due compagni appaiono ai piedi di Efrem, severi e rattristati, entrambi con il bastone ${ }^{51}$. Sullo sfondo vi sono alcune alture, con o senza vegetazione, e un edificio forse da interpretare come una chiesa. Efrem, nato all'inizio del IV secolo a Nisibi (Mesopotamia), è ricordato per la sua ricca produzione letteraria (sermoni, inni e scritti esegetici) e la sua conoscenza teologica; spende gli ultimi anni di vita a Edessa; unisce la vita contemplativa all'azione apostolica e muore nel $373^{52}$. Negli Atti siriaci leggiamo che "alle sue esequie concorsero tutti dalla città e luoghi vicini. Secondo il suo volere, il suo cadavere fu rivestito della sola tunica e tumulato nel cimitero dei forestieri; senonché, dopo poco tempo, i cittadini lo tolsero di lì e lo seppellirono nella grotta ove si tumulavano i vescovi della città" ${ }^{3}$. Dunque Efrem non voleva una ricca sepoltura e nel Menologio di Basilio II è rappresentata, in accordo con queste volontà narrate anche nella sua Vita metafrastica, una scena molto

divergenza dei testi in collegamento con la visione della croce che compare o è suggerita in entrambe le miniature (ma è giustificata solo nel codice W. 521 che riporta la versione del Metafrasta nella quale l'episodio miracoloso è menzionato). In merito a questa epifania cfr. anche V. RUGGIERI, A. ACCONCI, J.M. FEATHERSTONE, op. cit. (n. 41), p. 8o.

${ }^{45}$ Oltre un secolo fa Gabriel Millet affermava che i modelli per l'apparato figurativo del Menologio di Basilio II risalivano a Menologia locali o icone di santi prodotte nelle varie località dove essi erano venerati: G. MILLET, L'art byzantin, in A. MICHEL, Histoire de l'art depuis les premiers temps chrétiens jusqu’à nos jours, I.I, Paris, 1905, p. 127-301, in part. p. 238. Non affronto, per motivi di spazio, questo spinoso problema dei modelli utilizzati dai miniatori del Menologio vaticano, questione per la quale rimando a ciò che icasticamente scriveva in merito Ihor Ševčenko: "There is no end to conjectures" (I. ŠEVČENKO, The Illuminators of the Menologium of Basil II, in Dumbarton Oaks Papers, 16, 1962, p. 243-276 con 19 figg., n. 103 a p. 275, cfr. inoltre n. 24 a p. 248).

${ }^{46}$ P. Franchi De’ Cavalieri (a cura di), op. cit. (n. 8), p. 89. Così si legge anche nel testo del manoscritto vaticano (Vat. gr. 1613, p. 330).

${ }^{47}$ Ringrazio Nicola Busino che mi ha indotto a riflettere su questa costruzione. In uno studio dedicato all'architettura sepolcrale bizantina in Lycia leggiamo che alcune tombe "hanno conservato a terra una ristretta area delimitata da una forma semicircolare in muratura" (A. FILIPOVIÖ, L'architettura sepolcrale bizantina sulle isole di Gemile e Karacaören in Lycia, in Orientalia Christiana Periodica, 78, 2012, p. 439-466, in part. p. 465), forma che sembra evocata nella nostra miniatura dal muro curvo che si vede a destra. All'inizio del XX secolo una costruzione a forma di tholos, con un'abside verso est e somigliante a una chiesa si trovava a circa un'ora e mezza dalla città di Milasa verso sud ed era stata da taluni identificata con la tomba di Eusebia-Xena che, secondo il suo bios, era stata edificata appunto a sud della città [V. RUGGIERI, A. ACCONCI, J. M. FEATHERSTONE, op. cit. (n. 41), p. 41-42, 71]. Questa costruzione potrebbe rappresentare in generale il modello di una tomba bizantina della Caria, benché non escludo che riproduca proprio la tomba di Eusebia. D'altronde è noto che le architetture, numerose, che compaiono nelle miniature del lussuoso manoscritto vaticano debbano in alcuni casi considerarsi riproduzioni, abbreviate ma riconoscibili, di reali complessi monumentali. Su questo tema mi limito a menzionare C. BARSANTI, Le architetture «ad limitem» del Menologio di Basilio II (Cod. Vat. Greco 1613) e la miniatura con la commemorazione del patriarca Ignazio, in Commentari, 28, 1977, 1-3, p. 3-25. ${ }^{48}$ V. RUGGIERI, A. ACCONCI, J.M. FEATHERSTONE, op. cit. (n. 41), p. 68 e 71 rispettivamente per le citazioni; inoltre p. 73, 79, 86.

${ }^{49}$ Walter, anni fa, proponeva questa classificazione: "For death there are, it seems, six generic iconographical types: the last illness, the lying in state (prothesis), transportation of the mortal remains, deposition in tomb or charnel-house, the mortal remains lying in their place of rest, cult at the sealed sarcophagus". Vedi C. WALTER, op. cit. (n. 5), p. 115.

${ }^{50}$ Commemorato il 28 gennaio. Per una descrizione sintetica della miniatura: P. FRANCHI DE' CAVALIERI, op. cit. (n. 8), p. 96.

${ }^{51}$ Probabilmente è nel giusto $C$. Walter quando scrive che i funerali di Efrem assurgono, più in generale, a modello per le esequie di un monaco santificato. C. WALTER, op. cit. (n. 5), p. 12o. La rappresentazione di sant'Efrem siro, come indicato da Svetlana Tomekovič, ècambiata nel tempo; questa miniatura ne presenta l'immagine iniziale: testa priva di copricapo, capelli corti e bianchi, fronte scoperta, barba bianca: S. TOMEKOVIĆ, Le «portrait» dans l'art byzantin: exemple d'effigies de moines du Ménologe de Basile II à Dečani, in Dečani et l'art byzantin au milieu du XIVe siècle, à l'occasion de la celebration de 650 ans du Monastere de Dečani, September 1985, Reçu à la IXe séance de la Classe des sciences historiques le 25 novembre 1987, rédacteur V.J. Djurić, Beograd, 1989 (Academie serbe des sciences et des arts. Colloques scientifiques, XLIX, Classe des sciences historiques, 13), p. 121-136, con 23 figg., in part. p. 125 e n. 29. Il 'ritratto' del santo su un'icona del Sinai datata tra VIII eX secolo, ma più probabilmente della seconda metà del X, è prossimo, secondo la studiosa, alla miniatura: Ibid., p. 128-129, fig. 15. ${ }_{52}$ J.-M. SAUGET, s.v., Efrem, siro, diacono, Dottore della Chiesa, santo, in Bibliotheca Sanctorum, IV, Roma, 1964, col. 944-949; F. RILLIET, s.v., Efrem Siro, in Nuovo dizionario patristico e di antichità cristiane, I, A-E, Genova-Milano, 2006, col. 1586-1590.

${ }_{53}$ G. RICCIOTTI, Sant'Efrem siro. Biografia - Scritti - Teologia, Torino-Roma, 1925, p. 26-44, in part. p. 38, che segue l'edizione siriaca del Lamy. Cfr. anche Ibid., p. 88. 
semplice ${ }^{54}$ : anche qui si privilegia la prothesis del corpo e non una solenne e affollata processione funebre. Con questo ovviamente non voglio affermare che l'iconografia sia una semplice 'traduzione' del testo letterario e, del resto, nello stesso Menologio di Basilio II, come è già stato sottolineato, vi sono illustrazioni che non seguono pedissequamente la narrazione agiografica ${ }^{55}$. Efrem aveva chiesto che non venissero usate candele, pur menzionate in altre fonti, oppure aromi (sebbene l'uso dell'incenso nei funerali fosse prescritto e qui, come nelle esequie di sant'Amun abate, troviamo un monaco intento ad agitare il turibolo) e che il suo corpo fosse deposto non nella porpora e nella seta ma nella sua tunica e nel suo mantello, insieme con gli stranieri, proibendo una sepoltura in chiesa ${ }^{56}$.

John R. Martin, nel lontano 1954, sottolineava come questa miniatura del codice vaticano costituisse un esempio dell'iconografia dei funerali di un monaco che nell'arte bizantina segue un tipo standard. La composizione dipinta sul manoscritto datato 1081 di Princeton contenente la Scala del Paradiso di Giovanni Climaco, relativa al gradino VI che ha per soggetto il 'Ricordo della morte' (Princeton, University Library, Garrett 16, f. 63v) è essenzialmente la stessa, seppure rovesciata (fig. 8): con i dolenti, la stuoia, il monaco incappucciato che fa uscire fumo dall'incensiere ${ }^{57}$; tuttavia il particolare dell'angelo che accoglie l'anima del trapassato che fuoriesce dalla bocca è assente nell'immagine del Menologio di Basilio II (si tratta di un motivo invece impiegato nei Salteri cosiddetti monastici $)^{58}$.

Successivamente, a partire dal XIV secolo ${ }^{59}$, le raffigurazioni delle esequie di Efrem diventeranno sempre più ricche di dettagli, seguendo, secondo Martin, in parte una fonte a noi nota, gli Atti siriaci, e in parte un testo non pervenuto ${ }^{60}$. Mentre Giovanni Gaetano Bottari nel Settecento proponeva il collegamento con numerose altre fonti ${ }^{6}$. Di recente Alessandra Malquori ha sostenuto che gli Atti, per quanto siano

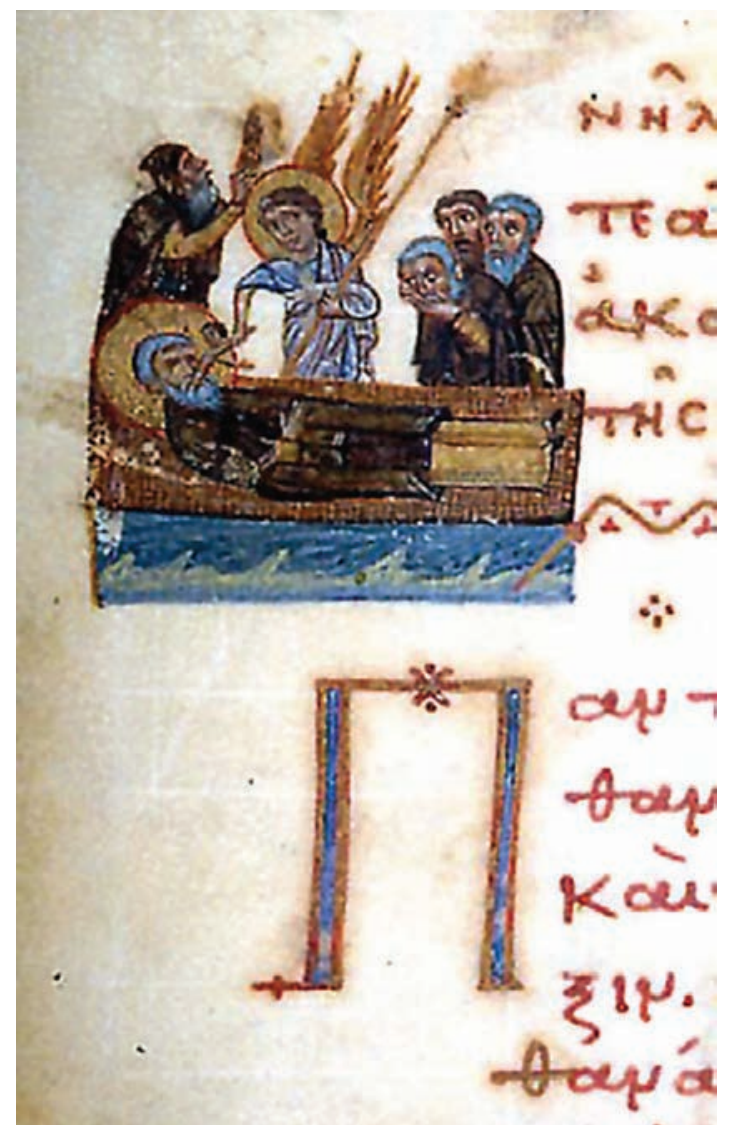

Fig. 8. Princeton, University Library, Garrett 16, f. 63v: Koimesis di un santo monaco.

una fonte pertinente, costituiscano un riferimento generico, limitandosi a narrare il giungere all'evento di monaci anacoreti, stiliti e cenobiti ${ }^{2}$, tra angeli e vigilanti, clero e popolo, col feretro accompagnato da inni e salmi ${ }^{63}$.

54 J.R. MARTIN, The Death of Ephraim in Byzantine and Early Italian Painting, in The Art Bulletin, 33, 4, 1951, p. 217-225, in part. p. 218.

55 Eे, ad esempio, il caso del martirio di san Bacco che avvenne per decapitazione: la miniatura che lo rappresenta (Vat. gr. 1613, p. 253) non segue fedelmente la leggenda agiografica e il monaco, di mezza età, viene rappresentato inclinato ma in piedi mentre riceve il colpo mortale, contrariamente alla notizia fornita dal testo agiografico che lo descrive in ginocchio nel momento cruciale, così come sottolineato da A. RIGO, «O martiri di Cristo apparsi di recente ...», in S. Gentile (a cura di), Oriente cristiano e santità. Figure e storie di santi tra Bisanzio e l'Occidente, cat. mostra (Venezia, Biblioteca Nazionale Marciana, 2 luglio-14 novembre 1998), [Milano], 1998, p. 15-34, in part. p. 24 e fig. 1 a p. 125.

${ }^{56}$ Così si legge nel suo testamento greco: J.S. ASSEMANI, Sancti Ephraem Syri opera omnia, ..., II, Roma, 1743, p. 230-247. Del testamento di Efrem si conservano due recensioni, una siriaca e una greca, "non scevre di qualche elemento leggendario": I. ORTIZ DE URBINA, s.v., Efrem, santo, in Enciclopedia Cattolica, III, Firenze, 1950, col. 126-129, in part. col. 126. Cfr. anche E. PERNIOLA, Sant'Efrem Siro. Dottore della Chiesa e Cantore di Maria, Santeramo in Colle (Bari), 1989, p. 25-29, in part. p. 27.

57 J.R. MARTIN, The Illustrations of the Heavenly Ladder of John Climacus, Princeton, 1954 (Studies in Manuscript Illumination, 5), p. 28-29, fig. 36. Sul manoscritto di Princeton mi limito a rimandare a Ibid., p. 24-47, figg. 29-66; S. KOTZABASSI, N. PATTERSON ŠEVČENKO, with collaboration of Don C. SKEMER, Greek Manuscripts at Princeton, Sixth to Nineteenth Century: A Descriptive Catalogue, Princeton, 2010, p. 112-125, figg. 127-170; B. PENTCHEVA, The aesthetics of landscape and icon at Sinai, in Res: Anthropology and Aesthetics, 65-66, 2014-15, p. 194-211, in part. p. 207-208, figg. 9-10. Il manoscritto è completato nel 1081 da uno scriba di nome Giuseppe. Questo codice, il più antico testimone illustrato contenente il testo di Climaco (almeno tra quelli conservati), mostra una stretta collaborazione tra lo scriba e il pittore: Ibid., p. 208.

${ }^{58}$ Vedi, ad esempio, il f. 137r del Salterio di Teodoro, conservato a Londra e datato 1066: S. DER NERSESSIAN, L'illustration des psautiers grecs du Moyen Âge, II. Londres, Add. 19.352, préface par A. Grabar, Paris, 1970 (Bibliothèque des Cahiers Archéologiques, 5), pl. 79, fig. 220. Per un approccio 'semiotico' al tema mi limito a rimandare a M. LEONE, Semiotica dellanima, in M. Leone, I. Pezzini (a cura di), Semiotica delle soggettività, Roma, 2013, p. 57-104. ${ }_{59}$ Cfr., in ultimo, M. DE GIORGI, op. cit. (n. 5).

${ }^{60}$ J.R. MARTIN op. cit. (n. 54), p. 218; J.R. MARTIN op. cit. (n. 57), p. 124-125; A. MALQUORI, La "Tebaide" degli Uffizi. Tradizioni letterarie e figurative per l'interpretazione di un tema iconografico, in I Tatti Studies. Essays in the Renaissance, 9, 2001, p. 119-137, in part. p. 129-131; M. DE GIORGI, op. cit. (n. 5), p. 195. ${ }^{61}$ G.G. BOTTARI, Sculture e pitture sagre estratte dai cimiterj di Roma pubblicate già dagli autori della Roma sotterranea e ora nuovamente date alla luce, III, Roma, 1754, p. 219-236. Martin scriveva: "He [cioè Bottari] was, it appears, not acquainted with the Syriac Acts of Ephraim, which supply details lacking in the Greek accounts (and which had been published, in Assemani's edition, about a decade earlier). For the scenes of cave-dwellers in the background Bottari sought parallels in the Apophthegmata, the Pratum spirituale of John Moschus, the Lausiac History of Palladius, and even in Theodoret's Religiosa historia; he did not, of course, entertain the notion, unheard of in his day, that a whole cycle of illustrations to one of these texts might have served as a model)": J.R. MARTIN op. cit. (n. 54), p. 223.

${ }^{62}$ A. MALQUORI, op. cit. (n. 6o), p. 129.

$6_{3}$ "Ejus corpus cum hymnis \& psalmis Spiritus Sancti, prosequentibus Angelis \& Vigilibus, Episcopis, \& Sacerdotibus, atque Diaconis, universoque Clero \& populo, ad tumulum elatum fuit. Convenere etiam ad parentalia laudabilis Chorus, \& benedictum agmen Anachoritarum, Stylitarum, \& Coenobitarum, a 


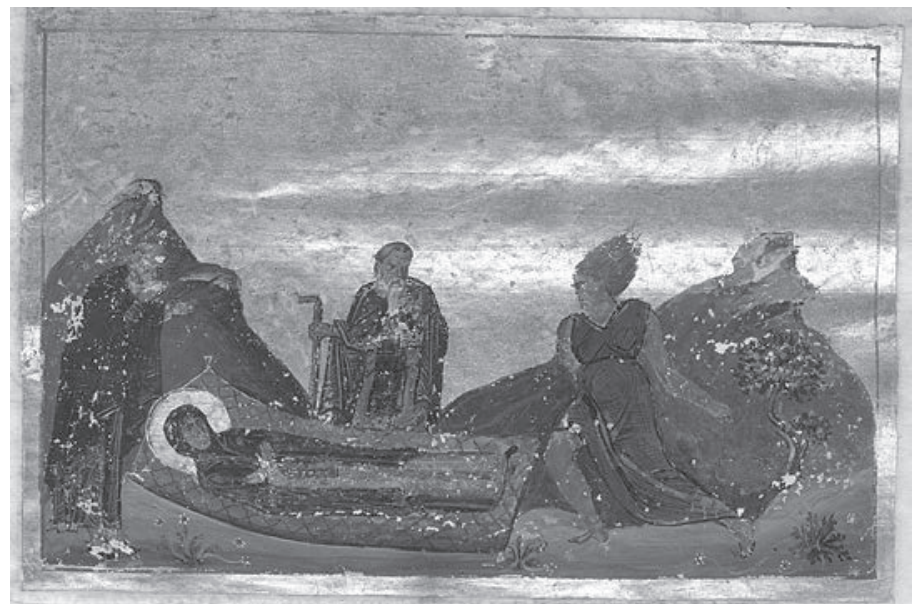

Fig. 9. Città del Vaticano, Biblioteca Apostolica Vaticana, Vat. gr. 1613, p. 394: Koimesis di santa Marina (C) 2017 per concessione della Biblioteca Apostolica Vaticana, ogni diritto riservato).

Santa Marina (o Maria) (Vat. gr. 1613, p. 394) ${ }^{64}$ visse in monastero, fingendosi uomo, con il nome di Marino ${ }^{65}$. Ė qui rappresentata giacente su un graticcio (fig. 9). Al suo capo un anziano monaco con barba alza le mani in gesto di meraviglia, un altro si trova dietro il cadavere, tiene un bastone in mano e ha un'espressione accigliata. Da destra avanza la donna che aveva accusato Marino di aver abusato di lei per giustificare la sua gravidanza: la calunniatrice è rappresentata come una menade con i capelli biondi sollevati in aria. Ancora una volta la scena si svolge all'aperto.

\section{IL DETTATO DELLE FONTI: ESEQUIE SENZA ONORI?}

Insomma, questa carrellata di immagini mostra la semplicità del rituale funebre in ambienti per lo più en plein air (Girolamo aveva scritto che Antonio, trovato morto Paolo, lo trasse fuori dalla spelonca e lo espose a cielo aperto cantando, in solitudine, i salmi secondo la tradizione cristiana $\left.{ }^{66}\right)$ : pochi confratelli (addirittura uno nel caso della Dormizione di Paolo) vegliano la salma distesa su una povera stuoia, eventualmente la incensano. Non ci sono candele, non c'è folla, né onori. Tutto ciò sembra corrispondere ad alcune indicazioni che possiamo leggere nelle fonti.

Come ha scritto Monique Alexandre: "La Vie d'Antoine, comme parfois d'autres Vies, comme certains textes de la tradition monastique, propose un modèle minimal de rites funéraires. Caché dans la terre, ignoré des hommes par le secret de son lieu de sépulture, Antoine, en sa mort, sera ce qu'il a été en sa vie: caché (§ 93). C'est dans 'la remémoration des saints', selon ses propres mots ( $§ 55)$, en un motif qui revient avec insistance autour de la mort d'Antoine, que l'homme de Dieu doit survivre parmi les hommes, à partir de l'écrit fixant les leçons de son ascèse et de sa mort. C'est là sa vraie gloire, après sa mort cachée, comme ce le fut déjà durant sa vie cachée (§ 93), sa vie d'anachorète. Tel est le culte des saints authentique" 67 .

Menziono inoltre un altro episodio che mi sembra significativo in tal senso ${ }^{68}$, lontano però nel tempo dagli esempi finora presentati e invece quasi contemporaneo all'epoca di esecuzione del manoscritto vaticano: siamo nel 1003, san Nilo (910-1004) si trova già da un po' a Serperi, vicino Gaeta, dove muore il beato Stefano, suo valoroso compagno; il santo dà allora l'ordine di preparare un doppio sepolcro perché i due potessero, arrivata la morte anche per lui, essere tumulati vicini; quando il duca di Gaeta lo viene a sapere dichiara la sua volontà di portare, giunto il trapasso per il santo, la salma di Nilo in città "perché questa lo abbia come fortissima torre", Nilo allora decide di trasferirsi in un luogo dove nessuno lo avrebbe riconosciuto, infatti "preferiva morire miseramente" ${ }^{69}$. Una fine della vita terrena senza onori, questo vuole il monaco vero.

Ovviamente abbiamo anche notizie di lunghe processioni funerarie con ceri e incensi, accompagnate dal canto dei salmi e da molta gente, per i santi monaci, vescovi e non ${ }^{70}$; ciò accade pure per onorare san Nilo, che muore nella chiesa di Sant'Agata a Tuscolo, "perché egli sempre ripeteva che «un monaco, salva forza maggiore, deve morire in chiesa»", ma viene sepolto, narra il suo bios, in un'"umile bara"71.

Già Christopher Walter, nel suo studio dedicato alla morte nell'arte bizantina, aveva sottolineato come il tema "particularly in monastic art, serve as a starting point for salutary meditation upon the vanity of human life, the awfulness of the Last Judgment and the timeliness of repentance. Such, indeed, Symeon of Thessalonika said, should be the normal content of sermons delivered at a funeral"72.

Nella tradizione liturgica bizantina, il più antico rituale per i funerali conosciuto è conservato in un manoscritto della Biblioteca Statale del Monumento Nazionale di Grotta-

quibus conditum fuit Ephraemi corpus cum pompa, \& honore Sanctorum in Caemeterio, sicut ipse Dei servus mandaverat" (Acta S.P.N. Ephraemi syriaca auctore anonymo Ex Codice Nitriensi Vaticano V, in S.E. ASSEMANI, S. Ephraem Syri opera omnia, III, Romae, 1743, p. XXIII-LXIII, in part. p. LXII). Brano già riportato in A. MALQUORI, op. cit. (n. 6o), p. 129. Assemani trascriveva da un codice vaticano, difatti questi "Atti siriaci, ci sono pervenuti in più di una recensione, quantunque in sostanza siano tutti somiglianti e dicano più o meno le stesse cose”: G. RICCIOTTI, op. cit. (n. 53), p. 27. Cfr. anche E. PERNIOLA, op. cit. (n. 56), p. 11.

${ }^{64}$ Commemorata il 12 febbraio. Per una descrizione sintetica della miniatura: P. FRANCHI DE' CAVALIERI, op. cit. (n. 8), p. 107.

${ }^{65}$ Le tradizioni (greca, latina, maronita ecc.) che si interessarono al culto di Marina ipotizzano la sua vita in luoghi differenti, difficile è anche stabilire la cronologia della sua esistenza, collocata da taluni nel V secolo: J.M. SAUGET, s.v., Marina (Maria)-Marino, monaca, santa, in Bibliotheca Sanctorum, VIII, Roma, 1966, col. 1165-1170; A. DI BERARDINO, s.v., Marina, in Nuovo dizionario patristico e di antichità cristiane, II, F-O, Genova-Milano, 2007, col. 3059. Cfr., inoltre, E. PATLAGEAN, L'histoire de la femme déguisée en moine et l'évolution de la sainteté féminine à Byzance, in Studi medievali, 17, 1976, p. 597623, in part. p. 604: in una lista di nomi di donne che sono divenute monache, la studiosa sottolinea che solo Marina I (rappresentata in questa miniatura), Pelagia, Matrona ed Eufrosine, sono commemorate nel Vat. gr. 1613. L'autrice non nomina santa Susanna e santa Eusebia romana.

${ }^{66}$ GIROLAMO, op. cit. (n. 1), p. 86.

${ }^{67}$ M. ALEXANDRE, op. cit. (n. 32), p. 270.

${ }^{68}$ Ringrazio vivamente Vera von Falkenhausen a cui devo questa segnalazione.

${ }^{69}$ Vita di S. Nilo fondatore e patrono di Grottaferrata, Versione e note a cura dello Jeromonaco G. Giovannelli, Grottaferrata, 1966, p. 110-111 (p. 111 per la citazione).

$7^{70}$ Per una breve lista che comprende però figure di santi non necessariamente monaci: M. ALEXANDRE, op. cit. (n. 32 ), n. 33 a p. 276.

${ }^{71}$ Vita di S. Nilo, op. cit. (n. 69), p. 116 e 117 .

${ }^{72}$ C. WALTER, op. cit. (n. 5), p. 127, e inoltre p. 117-118, 120-121. 
ferrata, Г. $\beta . X$, un Eucologio del X-XI secolo proveniente dal monastero di Carbone, in provincia di Poten$\mathrm{za}^{73}$. In esso il rito funebre appare lo stesso per tutti, con lievi differenze a seconda che si tratti di un laico o di un monaco (ad es. si recita il salmo 90 in questo caso quando il defunto è al centro della chiesa) e non è previsto uno speciale ordo per i preti ${ }^{74}$. Il primo testimone che differenzia il rito funebre tra monaci, fedeli e bambini è lo Schematologion della Biblioteca Regionale Universitaria di Messina, Mess. gr. 172, del 1178-7975.

Al contempo i documenti delle fondazioni monastiche bizantine (typika, testamenti e codicilli ecc.) forniscono fino all'XI secolo pochissime disposizioni per la sepoltura del monaco, ad esempio si indica il luogo o si ordina la commemorazione liturgica; peraltro queste disposizioni interessano per lo più solo il fondatore, nel cui giorno di morte si devono distribuire doni ${ }^{7}$. Nel XII secolo le cose cambieranno. E proprio in un manoscritto del XII secolo contenente la Scala del Paradiso di Giovanni Climaco, un testo fondamentale per il monaco, la "vanagloria" è rappresentata da un funerale (Sinait. gr. 418, f. 177r) (fig. 10) ${ }^{77}$.

\section{L'ABITO}

Nel Vat. gr. 1613 i monaci sono generalmente rappresentati, come scrive Anderson, con tunica, mandyas e analabos ${ }^{78}$, raramente hanno la testa coperta - ad esempio sant'Antonio nelle Koimeseis di Amun (p. 9o, fig. 3), di Paolo (p. 321, fig. 4) e nella sua (p. 327, fig. 5), anche il monaco intento a co-

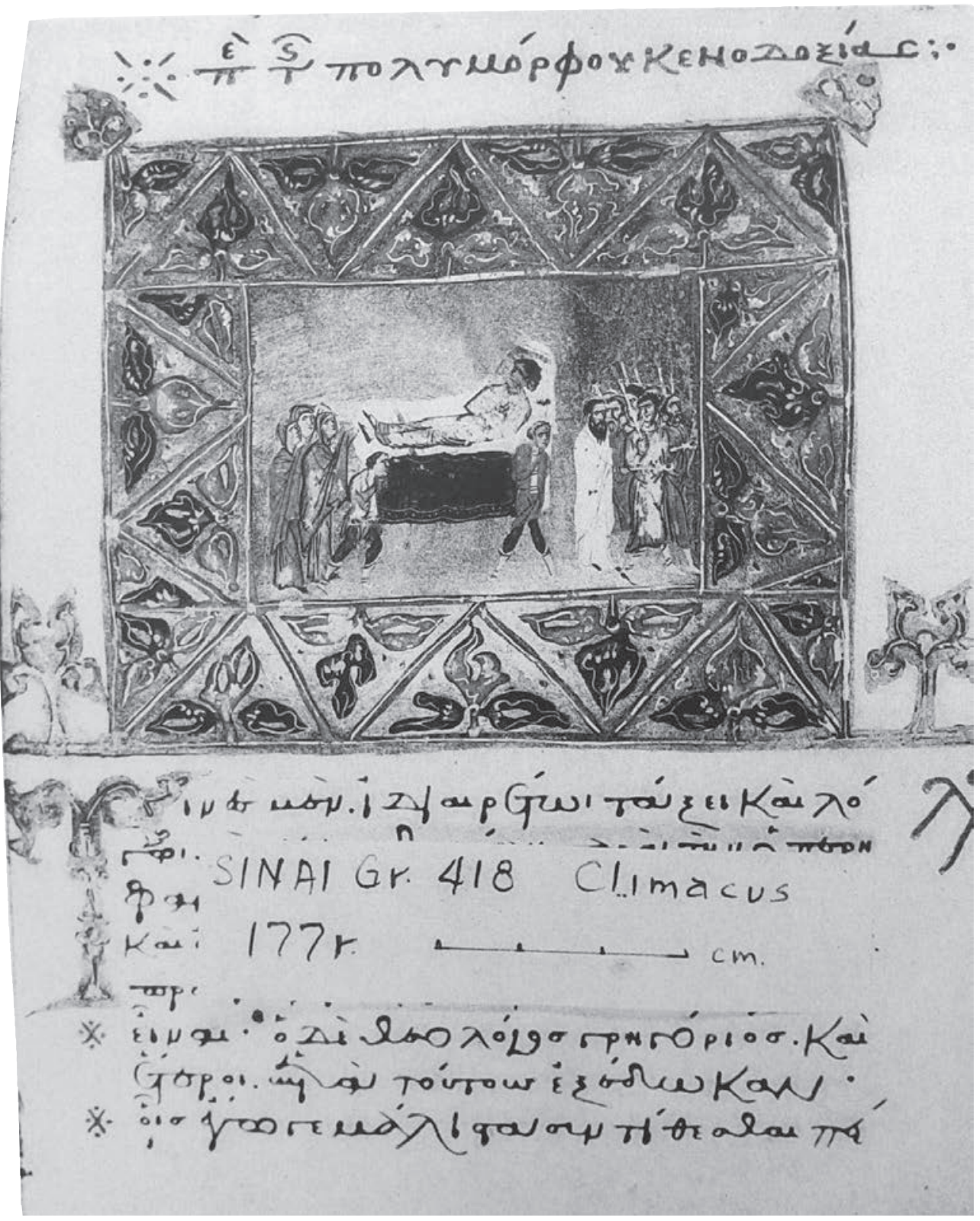

Fig. 10. Sinai, Monastero di Santa Caterina, Sinait. gr. 418, f. 177r: la vanagloria.

73 E. VELKOVSKA, Funeral Rites according to the Byzantine Liturgical Sources, in Dumbarton Oaks Papers, 55, 2001, p. 21-45, in part. p. 30-36. L'Eucologio è un libro liturgico che riporta i formulari eucaristici, le preghiere presidenziali della Liturgia delle ore, i riti dei sacramenti e benedizioni a preghiere per varie situazioni. ${ }^{74}$ Ibidem, p. 31.

75 Ibidem, p. 38-39. Lo Schematologion è un testo che contiene "almost exclusively not just the prayers but the entire ritual for the conferral of the monastic habit as well as for the funeral of a monk" (Ibid., p. 36).

${ }^{76}$ Il testamento di Apa Abraham, vescovo di Hermonthis, per il monastero di San Phoibammon vicino Tebe in Egitto (il convento di San Phoibammon è stato completamente sacrificato dal recupero del tempio faraonico all'inizio del Novecento: cfr. S. DONADONI, s.v., Copta, arte, in Enciclopedia dell'Arte Antica, classica e orientale, II, Roma, 1994, p. 280-283, in part. p. 281-282), databile al primo quarto del VII secolo, lascia alcune scarne istruzioni in merito alla questione: che il suo corpo venga avvolto e che si svolga la liturgia in suo onore secondo il costume del paese e le sue intenzioni. Cfr.: http://www.doaks. org/resources/publications/doaks-online-publications/byzantine-monastic-foundation-documents/typoo7.pdf (22 maggio 2016).

Il typikon di Timoteo per il monastero della Madre di Dio Evergetis fuori le mura di Costantinopoli, la cui prima versione risale agli anni 1054-70 e quella finale agli anni 1098-1118, riferisce solo che un prete deve prendersi cura delle sepolture, le morti devono essere commemorate liturgicamente e nel giorno della morte del monaco fondatore vanno distribuiti doni. Cfr.: http://www.doaks.org/resources/publications/doaks-online-publications/byzantine-monastic-foundation-documents/typo31.pdf (22 maggio 2016).

77 J.R. MARTIN op. cit. (n. 57), p. 96 e fig. 203.

${ }^{78}$ J.C. ANDERSON, The Illustrated Sermons of James the Monk: their Dates, Order, and Place in the History of Byzantine Art, in Viator. Medieval and Renaissance Studies, 22, 1991, p. 69-120, in part. n. 26 a p. 74. L'autore individua nel manoscritto vaticano una fonte importante e ricca d'informazioni sul vestiario bizantino tra il 976 e il 1025 quando la moda stava in parte cambiando.

${ }^{79}$ G. CORNU, M. MARTINIANI-REBER, Splendeur des étoffes orientales. Le ménologe de Basile II, in L'oeil 1987, 381, p. 24-29, in part. p. 29, fig. 16: si tratta della "petite cape en demi-cercle, le koukkoulion des moines byzantins" che ancora oggi indossano, scrivono le due autrici, i monaci siriani, armeni e copti; questo copricapo venne adottato dai musulmani e lo indossarono califfi e persone devote e importanti, è designato nei testi arabi del IX e X secolo con il 


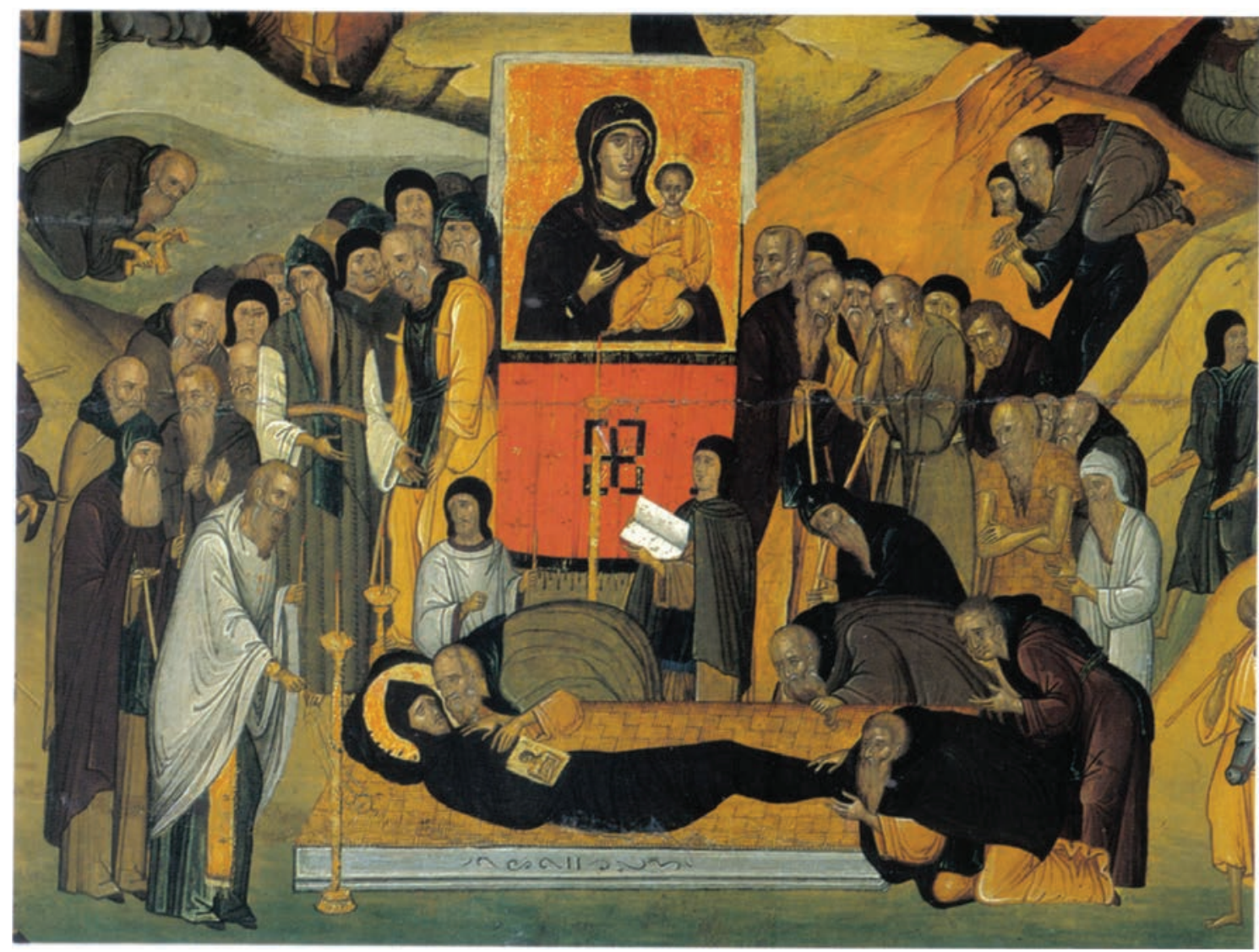

Fig. 11. Atene, Museo Bizantino e Cristiano, icona, part.: Koimesis di sant'Efrem siro (da M. De Giorgi 2013).

con una sorta di koukoulion, o meglio una sua variante, come santa Eusebia romana (p. 330, fig. 6) ${ }^{80}$. Le scarpe nere sono indossate sul piede nudo e qualche volta sono decorate da un nastro o linguetta bianca ${ }^{81}$, solo in poche miniature il monaco può portare calzini bianchi (ad esempio, san Daniele stilita: p. 237 ${ }^{82}$. Nel Menologio vaticano però troviamo anche monaci a piedi nudi: sant'Amun e sant'Antonio nella miniatura che rappresenta la morte del primo (p. 9o, fig. 3) e sant'Efrem (p. 354, fig. 7) ${ }^{83}$. Sappiamo d'altronde dalle fonti che i monaci in Oriente solevano andare scalzi ma, per difendersi da una malattia, dal freddo o dal caldo eccessivi, calzavano sandali ${ }^{84}$, tuttavia non scarpe chiuse come invece sono rappresentate nel Menologio di Basilio II.

Nel suo testamento Efrem ordina di essere deposto con tunica e mantello ${ }^{85}$, e anche nella sua Vita scritta dal Metafrasta si legge che egli comandò di non essere sepolto con una veste ricca ${ }^{86}$. Così anche Paolo chiese ad Antonio di essere deposto con il mantello che il vescovo Atanasio aveva donato ad Antonio ${ }^{87}$ : esso "diventa il simbolo dell'appartenenza alla retta fede cattolica, professata e difesa da sant'Atanasio" ${ }^{\prime 8}$.

nome di taylasān. Cfr. anche G. CORNU, M. MARTINIANI-REBER, Etoffes et vêtements dans le Ménologe de Basile II, reflets des courants d'échanges entre Byzance et le monde islamique, in Quaderni di studi arabi, 15, 1997, p. 45-64, in part. p. 56: le due studiose puntualizzano che tale copricapo è attualmente indossato dai preti copti e assiri, e il velo del clero armeno e ortodosso deriva proprio da questa particolare copertura del capo; nella miniatura con sant'Efrem troviamo un tipo di copricapo, una sorta di piccola mantellina semi-circolare che si mette sul berretto o sul turbante: essa è portata dalla gente rispettabile, dagli uomini pii, dai sapienti e qui lo indossa il personaggio con l'incensiere che veglia il santo morto. Cfr. inoltre S. TOMEKOVIĆ, Formation de l'iconographie monastique orientale (VIII -Xe siècles), in Revue bénédictine, 103, 1993, p. 131-152, in part. n. 67 a p. 143. L'autrice di questo studio sottolinea come i monaci compaiano, nel manoscritto in questione, a capo scoperto, e solo in pochi casi figurino con un cappuccio sulla testa (Vat. gr. 1613, p. 2, 90, 237-238, 321, 327). Mentre Cassiano scriveva "nello stesso vestito dei monaci d'Egitto si notano alcuni espedienti destinati non tanto a una maggior cura del corpo, quanto piuttosto a dar rilievo al loro genere di vita: viene così assicurata, nel loro stesso adattamento dell'abito, la pratica della semplicità e dell'innocenza. Usano infatti dei cappucci molto piccoli (cuculli), che essi distendono e abbassano giù fino al collo e alle spalle, in modo però da coprire soltanto il capo; ne fanno uso continuamente, di giorno e di notte, proprio a questo fine, per essere indotti a custodire con perseveranza l'innocenza e la semplicità dei fanciulli, di cui quel copricapo è in qualche modo un'imitazione”. Cfr. GIOVANNI CASSIANO, op.cit. (n. 15), p. 61 (Libro I.3: "L'uso del copricapo, come simbolo d'innocenza").

${ }^{80}$ J.C. ANDERSON, op. cit. (n. 78), nn. 26, 28-29 alle p. 74-75. Agli esempi menzionati in questo studio possiamo aggiungere altri casi.

${ }^{8}$ S. TOMEKOVIĆ, op. cit. (n. 79), p. 148 e nn. 86-87.

${ }^{82}$ Ibidem, n. 85 alla p. 148.

${ }^{8}$ Ibidem, p. 148.

${ }^{84}$ GIOVANNI CASSIANO, op.cit. (n. 15), p. 64-65 (Libro I.9). Franchi de' Cavalieri rimanda inoltre a Gregorio Nazianzeno e Girolamo: P. FRANCHI DE' CAVALIERI, op. cit. (n. 8), n. 2 a p. 25.

${ }^{85}$ J.S. ASSEMANI, op. cit. (n. 56), p. 234.

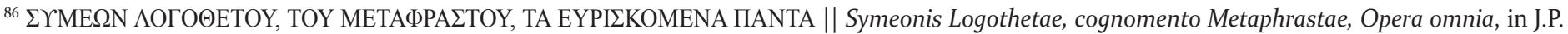
Migne (accurante), Patrologia graeca, CXIV, Tomus primus, Paris, 1864, col. 1253-1268, in part. col. 1267-1268.

${ }^{87}$ GIROLAMO, op. cit. (n. 1), p. 83-84 (12). Su questo mantello cfr. ATANASIO DI ALESSANDRIA, op. cit. (n. 17), p. 125 (91.8). La notizia si legge anche sul manoscritto vaticano (Vat. gr. 1613, p. 321).

${ }^{88}$ GIROLAMO, op. cit. (n. 1), n. 6 a p. 84 . 


\section{LA VALORIZZAZIONE DELLUMILTÀ E DELL'ASCESI}

Le scene finora osservate, di estrema semplicità, collocate in un paesaggio per lo più spoglio, enfatizzano l'ascesi del monaco che, morto e rappresentato su un'umile stuoia o direttamente sul terreno, tiene sempre le mani incrociate al petto, gesto che significa sottomissione, umiltà, reverenza. Abbinate alla posizione supina le braccia così sistemate indicano il defunto e in questo contesto il gesto ricorre in molte tradizioni figurative ${ }^{89}$. Nei casi che qui stiamo analizzando potrebbe rimandare al tema dell'umiltà, decisamente importante per il monaco bizantino, a cui Giovanni Climaco dedica, nella sua Scala, il gradino XXV ${ }^{\circ}$. Il gesto è lo stesso dell'Imago pietatis, iconografia di origine bizantina che però con questa variante delle braccia incrociate compare in Oriente non prima della fine del XIII-inizi XIV secolo ${ }^{91}$.

Il termine Akra Tapeinosis (somma umiliazione) è spesso riferito a quest'immagine, ma non si conoscono né la fonte, né la connessione con l'iconografia; il termine non compare in Isaia 53,4 (che usa solo la parola tapeinosei), né è connesso a un'immagine esistente ${ }^{92}$. Indubbiamente l'iconografia in questione meriterebbe ulteriori studi. Ciò che però qui ci interessa è che in alcune Dormizioni del monaco può comparire sul petto del defunto proprio l'icona dell'Akra Tapeinosis (la testimonianza più antica sembrerebbe essere la pittura murale nella chiesa di San Giovanni a Koudoumas, Creta, risalente forse al 136o, seppure rimangano alcuni dubbi sul soggetto della tavola appoggiata sul petto; in ogni caso si tratta sempre di testimonianze cronologicamente tarde: fig. 11, icona della seconda metà del Quattrocento) ${ }^{93}$. La critica si è chiesta il motivo di questa presenza e l'ha connessa alla valenza liturgico-funeraria dell'immagine, seppure la scelta di questo soggetto, al posto di altri (icona del Pantokrator) non appaia tassativa ${ }^{94}$. Mi domando, ma ulteriori ricerche andrebbero condotte in questa direzione, se anche l'umiltà del monaco non possa aver influito (e forse la mancanza di una fonte specifica ha reso la scelta del soggetto sull'icona arbitraria). Ricordo che un insegnamento tipico degli Apoftegmi consiste nel considerare se stessi un nulla, nell'ottica dei Padri del deserto nell'essere quindi umili95.

\section{LA NASCITA DELL'ICONOGRAFIA E LA QUESTIONE DEI MODELLI ALTI}

La critica ha sottolineato, nella genesi iconografica della Koimesis del monaco, la dipendenza - o piuttosto la derivazione - da modelli di Dormizioni ben più consuete, in primis quella della Vergine, iconografia nata in età preiconoclasta ma diffusasi solo a partire dal X-XI secolo ${ }^{96}$ (fig. 12: avorio datato alla metà del X secolo), e a seguire le Koimeseis di santi, per lo più vescovi ${ }^{97}$. Le immagini qui prese in considerazione, che vedono coinvolto l'umile monaco, presentano però alcune differenze rispetto ai modelli per così dire 'aulici' ora menzionati, differenze che non è opportuno trascurare, naturalmente dando conto anche delle affinità.

Puntualizzo che non sono giunte sino a noi, per quanto io ne sappia, testimonianze di Dormizioni "monastiche" precedenti al X o meglio XI secolo (escludendo però, nuovamente, i monaci divenuti vescovi), immagini non lontane, almeno dal punto di vista compositivo, dai primi esempi iconografici

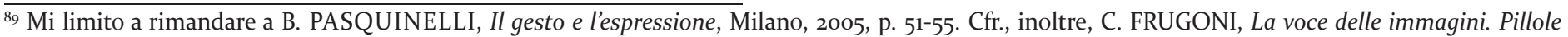
iconografiche dal Medioevo, Torino, 2010, p. 9-14.

$9^{\circ}$ GIOVANNI CLIMACO, op. cit. (n. 24), p. 418-434.

${ }^{91}$ L'iconografia, di cui Hans Belting ha confermato l'origine bizantina, è dell'XI secolo, anche se la prima testimonianza nota è del XII secolo (icona su due lati al Museo Bizantino di Kastoria). Cfr. almeno H. BELTING, An Image and its Function in the Liturgy: the Man of Sorrows in Byzantium, in Dumbarton Oaks Papers 34-35, 1981, p. 1-16. E, per una sintesi, N. PATTERSON ŠEVČENKO, s.v., Man of Sorrows, in The Oxford Dictionary of Byzantium, II, New York-Oxford, 1991, p. 1287.

${ }^{92}$ K. CORRIGAN, N. PATTERSON ŠEVČENKO, 'The teaching of the ladder': The Message of the Heavenly Ladder Image in Sinai ms. gr. 417 , in Images of the Byzantine World: Visions, Messages and Meanings: Studies presented to Leslie Brubaker, Farnham-Surrey-Burlington, 2011, p. 99-120, in part. n. 32 a p. 113. ${ }_{93}$ M. DE GIORGI, op. cit. (n. 5), p. 190-191. Ancora oggi sul corpo del monaco defunto si adagia l'icona di Cristo. D’altronde Simeone, arcivescovo di Tessalonica (1416/17-29), nel De ordine sepolturae scriveva, come ricorda M. De Giorgi, "Sacra Christi imago super cadavere defuncti ponantur" (Ibid., p. 191). ${ }_{94}$ Ibidem, p. 191. L'immagine sarebbe legata, secondo Belting, al servizio della liturgia della Settimana Santa, e quindi sarebbe da connettere alla passione e morte di Cristo: H. BELTING, op. cit. (n. 91), p. 3 e ss. Mi permetto di sottolineare che alcuni elementi rituali della cerimonia funebre (tanto del monaco quanto del laico, e già risalenti al X-XI secolo) "offer an implicit paschal perspective": E. VELKOVSKA, op. cit. (n. 73), p. 44-45.

${ }^{95}$ GIOVANNI CLIMACO, op. cit. (n. 24), n. 1 a p. 418.

${ }^{96} \mathrm{~T}$. VELMANS, La visione dell'invisibile. L'immagine bizantina o la trasfigurazione del reale. Lo spazio, il tempo, gli uomini, la morte, le dottrine, Milano, 2009 (Di fronte e attraverso, 846 - Storia dell'arte, 40), p. 77-78: "si immaginò la morte di Maria, basandosi sugli apocrifi e sulle omelie riguardanti la dormizione della Vergine di san Giovanni Damasceno. Se l'immagine apparve nel VII-VIII secolo nella chiesa di Sion a Gerusalemme, essa cominciò a diffondersi solo nell'XI secolo e si sviluppò a partire dal XII; è solo alla fine del XIII secolo che la scena si trasforma talvolta in ciclo"; M. DE GIORGI, op. cit. (n. 5), nt. 7 a p. 198 (l'autrice rimanda a un suo contributo in corso di pubblicazione: Il Transito della Vergine. Testi e immagini dall'Oriente al Mezzogiorno medievale, Spoleto). Cfr. inoltre per le fonti: M. JUGIE, La Mort et l’Assomption de la Sainte Vierge. Étude historico-doctrinale, Città del Vaticano, 1944 (Studi e testi, 114); M. VAN ESBROECK, Aux origines de la Dormition de la Vierge. Études historiques sur les traditions orientales, Aldershot, 1995 (Collected Studies Series, 472). Mentre per una sintesi utile da un punto di vista artistico mi limito a menzionare: K. KREIDL-PAPADOPOULOS, Koimesis, in M. Restle, K. Wessel (hrsg. von), Reallexikon zur byzantinischen Kunst, IV, Stuttgart, 1966, p. 136-182; I. ZERVOU TOGNAZZI, L'iconografia della Koimisis della Santa Vergine, specchio del pensiero teologico dei Padri bizantini, in Studi e ricerche sull'Oriente cristiano, 8, 1985, p. 21-46, 69-9o (in questo studio Zervou Tognazzi afferma, come farà in seguito in altre sedi e per altri temi figurativi, che l'iconografia dipende dalle omelie patristiche e non dai testi apocrifi); R.F. TAFT, A. WEYL CARR, s.v., Dormition, in The Oxford Dictionary of Byzantium, I, New York-Oxford, 1991, p. 651-653; E. MARCATO, s.v., Maria. Area bizantina, in Enciclopedia dell'arte medievale, VIII, Roma, 1997, p. 227-234, in part. p. 232-233. Vedi anche, in particolare per il rapporto con altre Dormizioni, A. WEYL CARR, Popular imagery, in H.C. Evans, W.D. Wixom (ed.), The Glory of Byzantium. Art and Culture of the Middle Byzantine Era A.D. 843-1261, cat. of exhibition (New York, Metropolitan Museum of Art, from March 11 through July 6, 1997), New York, 1997, p. 113-117, 511-512, in part. p. 114-117. In ogni caso l'iconografia della Dormizione della Vergine a cui facciamo riferimento risale al X secolo. Queste prime versioni poi dovettero arricchirsi con il tempo (nella chiesa di Sion, secondo la testimonianza di Beda il Venerabile, compariva Maria come una mummia e l'angelo con la palma). Ibidem, n. 22 a p. 512. ${ }_{97}$ Ibidem, p. 116-117; T. VELMANS, op. cit. (n. 96), p. 96: "Le rappresentazioni di cerimonie commemorative per i laici sono composte secondo gli schemi usati per raffigurare la dormizione dei santi, a loro volta influenzati da quello della dormizione della Vergine”; M. DE GIORGI, op. cit. (n. 5), p. 189.
} 


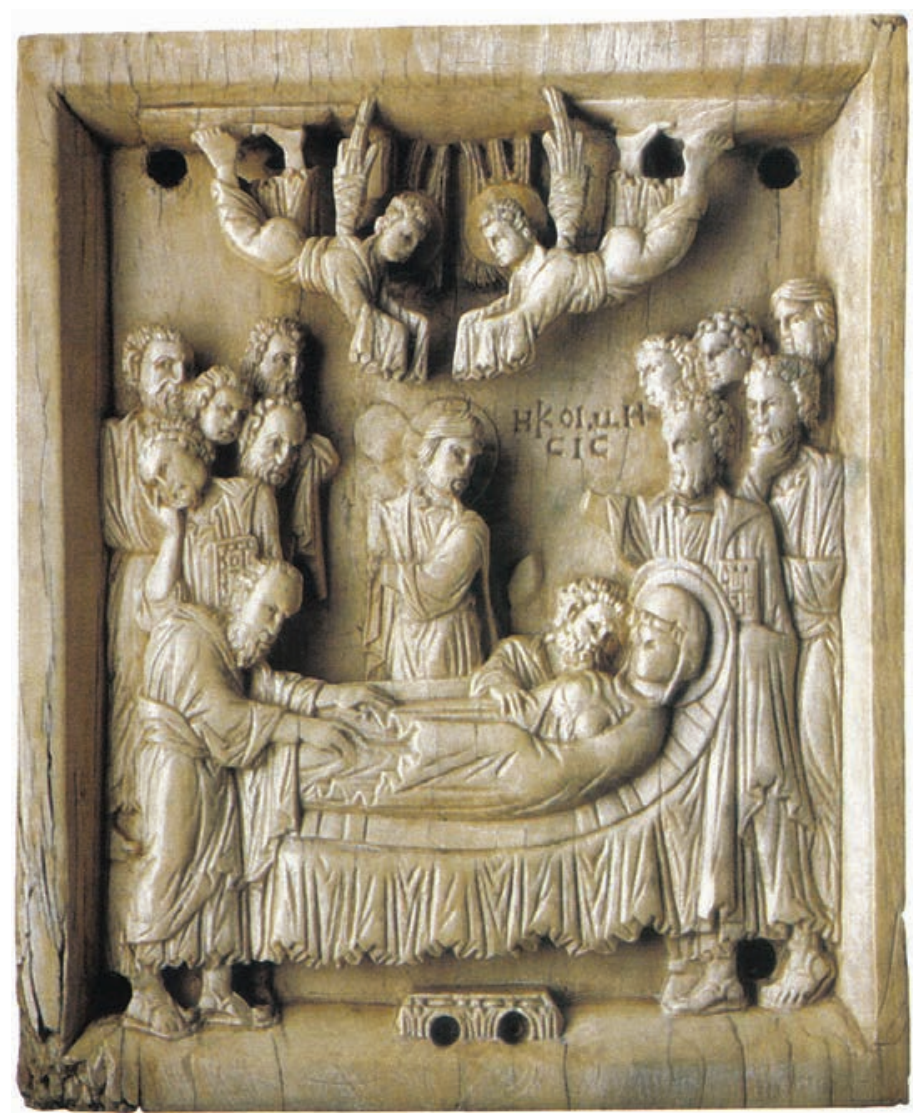

Fig. 12. Houston, The Museum of Fine Arts, avorio: Koimesis della Vergine.

che immagino perduti $i^{98}$. Affermo questo valutando anche la semplicità della scena, che poi si complica, ne è testimone Efrem ma il discorso si ripete per Saba, Eutimio e altri.

Nelle scene miniate di XI secolo che rappresentano il tema in questione mancano alcuni elementi fondamentali presenti nella Koimesis della Vergine (fig. 12) come la figura di Cristo con l'animula della defunta, gli Apostoli (in genere dolenti) e il ricco catafalco ${ }^{99}$, ma rimane la salma distesa, eventualmente compare una figura con l'incensiere, l'animula portata in cielo (non da Cristo però) e il bacio ai piedi. In queste prime immagini, le braccia incrociate al petto caratterizzano in genere (ovviamente vi sono eccezioni) la figura del monaco, mentre nel caso della Vergine possono comparire ma anche essere disposte lungo il corpo.

Qualora appaia, nelle scene di ufficio funebre del monaco, un personaggio con il turibolo, questo è sempre rappresentato al capo del defunto, così come avviene nella Dormizione della Vergine: infatti in questa iconografia è Pietro che tiene l'incensiere in mano, per indicare che è lui a recitare la preghiera, e, dal momento che è anche capo della Chiesa, allora lo si colloca presso il capo della Vergine che è simbolo della Chiesa, come afferma Ioanna Zervou Tognazzi leggendo la terza omelia in Dormitionem B. Mariae del patriarca Germano $(† 733)^{100}$. Questo particolare sembrerebbe indirizzare, effettivamente, verso una dipendenza della Koimesis del monaco (dove nei primi esempi analizzati la posizione del turibolante è sempre al capo del defunto) da quella della Vergine.

Riterrei invece poco probabile la derivazione, a una cronologia così alta, dalle Dormizioni dei vescovi. In tali casi la scena è caratterizzata da ricche lettighe, eventualmente ambienti urbani e candele (fig. 1), diversa, talvolta, la posizione del personaggio con il turibolo, mentre sul petto del vescovo può comparire il Vangelo (in uno Schematologion italo-greco dell'XI secolo leggiamo che se il monaco è un egumeno, un prete o un diacono, si pone sul petto del defunto appunto il Vangelo ${ }^{101}$. Questo libro, nelle prime testimonianze figurative, non compare mai invece sul monaco defunto che non ha fatto carriera, per così dire, all'interno della Chiesa, forse a sottolineare visivamente la sua distanza da una prospettiva ecclesiologica.

Probabilmente è lecito pensare, come già è stato scritto dalla Weyl Carr per la Koimesis della Vergine, a un comune passato pagano (una scena di Lamento funebre), assimilato e interpretato ovviamente in chiave cristiana ${ }^{102}$. Ricordo però, con le parole di Salvatore Settis, che somiglianza di schema (e a questa faccio riferimento) non significa derivazione ma "semmai solo una linea tradizionale di lungo periodo, che comincerebbe nell'antichità continuando a Bisanzio" ${ }^{103}$. Il resto - tranne piccole divergenze ${ }^{104}$ - mi sembra sia raccontato dalle fonti, a sottolineare, ancora una volta, la stretta

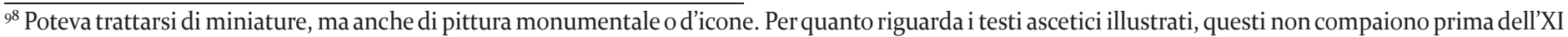
secolo, dobbiamo dunque cercarne un riflesso in altre opere come ad esempio i Sacra Parallela di san Giovanni Damasceno (675-750) (Paris, Bibliothèque nationale de France, cod. gr. 923) che mostrano, come affermava molto tempo fa John R. MARTIN (An Early Illustration of The Sayings of the Fathers, in The Art Bulletin, 32, 4, 1950, p. 291-295), che la letteratura ascetica era corredata da immagini nel IX secolo e forse anche in età preiconoclasta. Tuttavia nel caso del manoscritto parigino non compaiono scene di morte, dunque l'ipotesi di Martin, benché probabile, non aiuta la nostra ricerca. Nei primi secoli di nascita del monachesimo, come sottolinea Giuseppa Zanichelli, le fonti "sembrerebbero escludere la presenza nella biblioteca monastica vera e propria di codici sontuosi e certamente la produzione libraria cenobitica, come possiamo restituire dalle origini grazie alla ricostruzione di quella del Monastero Bianco, sembrerebbe non seguire la lussuosa tradizione alessandrina": G.Z. ZANICHELLI, I codici del deserto: immagine e visione, in B. Astrua (a cura di), Itinerari mediterranei fra IV e IX secolo. Città-capitale e Deserto-monastico, Atti del convegno (Genova, 11-13 novembre 2010), Torino, 2013, p. 105-120, in part. p. 110-111. In ogni caso, perlomeno a partire dal VI secolo, registriamo anche casi di produzione di libri illustrati per il monastero in area bizantina, e in seguito di egumeni ambiziosi che ordinano codici riccamente e significativamente miniati, come quel Michele Sincello, egumeno del monastero costantinopolitano di Studio, che fece realizzare nel 1066 il manoscritto oggi definito Salterio di Teodoro (Londra, British Library, Add. MS 19352), con immagini che lo ritraggono: in una, emblematica, egli appare investito della sua carica (rappresentata dal bastone) dal Pantokrator che si affaccia dalla volta celeste. Per il tema in generale (committenza monastica di libri illustrati) mi limito a rimandare a A. IACOBINI, Libri per i monaci. Segni e immagini di committenza monastica nel mondo bizantino, in Rivista di studi bizantini e neoellenici, nuova serie, 43, 2006, p. 3-19.

${ }_{99}$ D'altronde già Walter scriveva: “Generally the prothesis of a saint is represented more simply than that of the Virgin", cfr. C. WALTER, op. cit. (n. 5), p. 120. ${ }^{100}$ I. ZERVOU TOGNAZZI, op. cit. (n. 6o), p. 69-71 e in part. n. 115 a p. 71. Le Omelie di Germano sulla Koimesis della Vergine possono leggersi in Patrologia Graeca, XCVIII, col. 339-372 (Sermo I, col. 339-348; Sermo II, col. 348-358; Sermo III, col. 360-372).

${ }^{101}$ Grottaferrata, Г.ß.XLIII (la celebrazione funebre è divisa in tre parti: 1. nella cella, il corpo viene lavato e vestito; 2 . nella chiesa, il servizio funebre; 3 . al cimitero, il seppellimento; i tre momenti sono legati da processioni): E. VELKOVSKA, op. cit. (n. 73), p. 37. Cfr. M. DE GIORGI, op. cit. (n. 5), p. 191.

${ }^{102}$ Cfr. A. WEYL CARR 1997, op. cit. (n. 96), p. 115.

${ }^{103}$ S. SETTIS, Ars moriendi: Cristo e Meleagro, in Annali della Scuola Normale Superiore di Pisa, Classe di Lettere e Filosofia. Quaderni 1/2, Quarta Serie, 9/10, 2000, p. 145-170, in part. p. 149.

${ }^{104}$ In particolare in merito all'abbigliamento.
} 
connessione tra storia e arte. Tuttavia, mi sia concessa, una puntualizzazione: le fonti a cui ho fatto riferimento riportano quasi tutte, tranne rari casi, per lo più fatti e detti non di prima mano, cioè distanti cronologicamente dagli eventi narrati, così che è lecito pensare che contengano "una me- scolanza di testimonianze autentiche e di tradizioni nate posteriormente"105. In ogni caso resta la nostra interpretazione; non credo che un bizantino dell'XI secolo si ponesse tale problema. Piuttosto nell'immagine cercava un esempio da seguire o una storia da guardare e per cui commuoversi, eventualmente da raccontare di nuovo ${ }^{106}$.

${ }_{105}$ T. ORLANDI, Introduzione, in Vite di monaci copti, a cura di Id., traduzione di A. Campagnano e T. Orlandi, Roma, 1984 (Collana di testi patristici, 41), p. 5-34, in part. p. 13. Lo studioso sottolineava, in questo caso, il problema per gli Apophthegmata Patrum e le Vite di Pacomio, ma non solo.

${ }^{106}$ Senza toccare l'ampia e complessa questione, mi limito qui a menzionare lo studio di A. Iacobini sugli affreschi delle celle-oratorio di Bāwīt, per i quali sembra "lecito supporre che la loro destinazione travalicasse l'uso strettamente personale. Se infatti, a quanto pare, il monaco anziano che viveva nella cella-oratorio teneva presso di sé uno o più giovani discepoli che apprendevano da lui i fondamenti della vita ascetica e cenobitica, allora si potrebbe ipotizzare che gli affreschi di questi ambienti assolvessero, per gruppi molto ristretti, ad una funzione didattica, e che fossero utilizzati come «libri di testo» per compiere esercitazioni in materia di fede cristiana": A. IACOBINI, Visioni dipinte. Immagini della contemplazione negli affreschi di Bāwīt, Roma, 2000 (Studi di arte medievale, 6), in part. p. 216-220 (la citazione si legge alle p. 216-217). 